University of Louisville

ThinkIR: The University of Louisville's Institutional Repository

Electronic Theses and Dissertations

4-2019

\title{
A deep learning approach to detect diabetic retinopathy in fundus images.
}

Winston R. Furtado

University of Louisville

Follow this and additional works at: https://ir.library.louisville.edu/etd

Part of the Biomedical Engineering and Bioengineering Commons

\section{Recommended Citation}

Furtado, Winston R., "A deep learning approach to detect diabetic retinopathy in fundus images." (2019). Electronic Theses and Dissertations. Paper 3449.

https://doi.org/10.18297/etd/3449

This Master's Thesis is brought to you for free and open access by ThinkIR: The University of Louisville's Institutional Repository. It has been accepted for inclusion in Electronic Theses and Dissertations by an authorized administrator of ThinkIR: The University of Louisville's Institutional Repository. This title appears here courtesy of the author, who has retained all other copyrights. For more information, please contact thinkir@louisville.edu. 


\author{
Winston Furtado \\ B.Sc. in Bioengineering, University of Louisville, 2018
}

\author{
A Thesis \\ Submitted to the Faculty of the \\ J.B. Speed School of Engineering of the University of Louisville \\ in Partial Fulfillment of the Requirements \\ for the Degree
}

Master of Engineering in Bioengineering

Department of Bioengineering

University of Louisville

Louisville, Kentucky

April 2019 
Submitted by:

Winston Furtado

A Thesis Approved on

(Date)

by the Following Reading and Examination Committee:

Ayman El-Baz, Ph.D. Thesis Director

Adel Elmaghraby, Ph.D.

Guruprasad A. Giridharan, Ph.D. 


\section{ACKNOWLEDGMENTS}

I would like to thank my thesis advisor Dr. El-Baz for his support over the past year. His mentorship has had a positive impact on me as a student and as a researcher; it has truly been rewarding. To the rest of my thesis committee, Dr. Elmaghraby and Dr. Giridharan, thank you for being a part of my thesis committee and helping me to achieve a special milestone in my college career. Also, I would like to thank Dr. Ahmed Shalaby for his guidance and assisting me whenever I needed help. Finally, I would like to thank my family for helping me stay motivated and determined. 


\begin{abstract}
A DEEP LEARNING APPROACH TO DETECT DIABETIC RETINOPATHY IN FUNDUS IMAGES

Winston Furtado
\end{abstract}

April 2019

Background: Diabetic retinopathy is a disease caused due by complications of diabetes mellitus which can lead to blindness. About 33\% of the US population with diabetes also show symptoms for diabetes retinopathy. If not treated, diabetic retinopathy worsens over time by progressing through two main pathological stages of non-proliferative and proliferative and four clinical stages. While the diagnostic accuracy of detecting diabetic retinopathy through machine learning have shown to be successful for OCT images, the accuracy of ultra-widefield fundus images have yet to be fully reported. This paper describes a method to non-invasively detect and diagnose diabetic retinopathy from ultra-widefield fundus images.

Methods: A total of 62 graded-images were obtained from the Cleveland Clinic. A deep learning algorithm was developed to identify and extract features from the images. The algorithm was then simulated to classify the test images into one of three clinical classes. Data was collected on the accuracy and probability of the diagnosis/classification.

Results: The classification algorithm had an average accuracy that ranged from $92 \%$ to $97 \%$ for the training images and $50 \%$ for the test images. Confusion matrices were created to obtain statistical measures of performance such as sensitivity, false negative rate, precision, and the false discovery rate. The sensitivity decreased from $70 \%$ to $50 \%$ as the image size increased. The precision also decreased from $65 \%$ to $50 \%$ as the image size increased. Validation methods such as image normalization and transfer learning showed no improvement in classification accuracy.

Conclusion: This study demonstrates the potential for applying deep learning algorithms to classify ultra-widefield images. This study also demonstrates the need for doctors to further examine the diagnosis to account for false positives and/or misdiagnosis. Additionally, limitations and their impact on the simulation of the deep learning algorithm were explored.

Keywords: diabetic retinopathy; deep learning; $\mathrm{CNN}$; image classification; ultra-wide field imaging 


\section{TABLE OF CONTENTS}

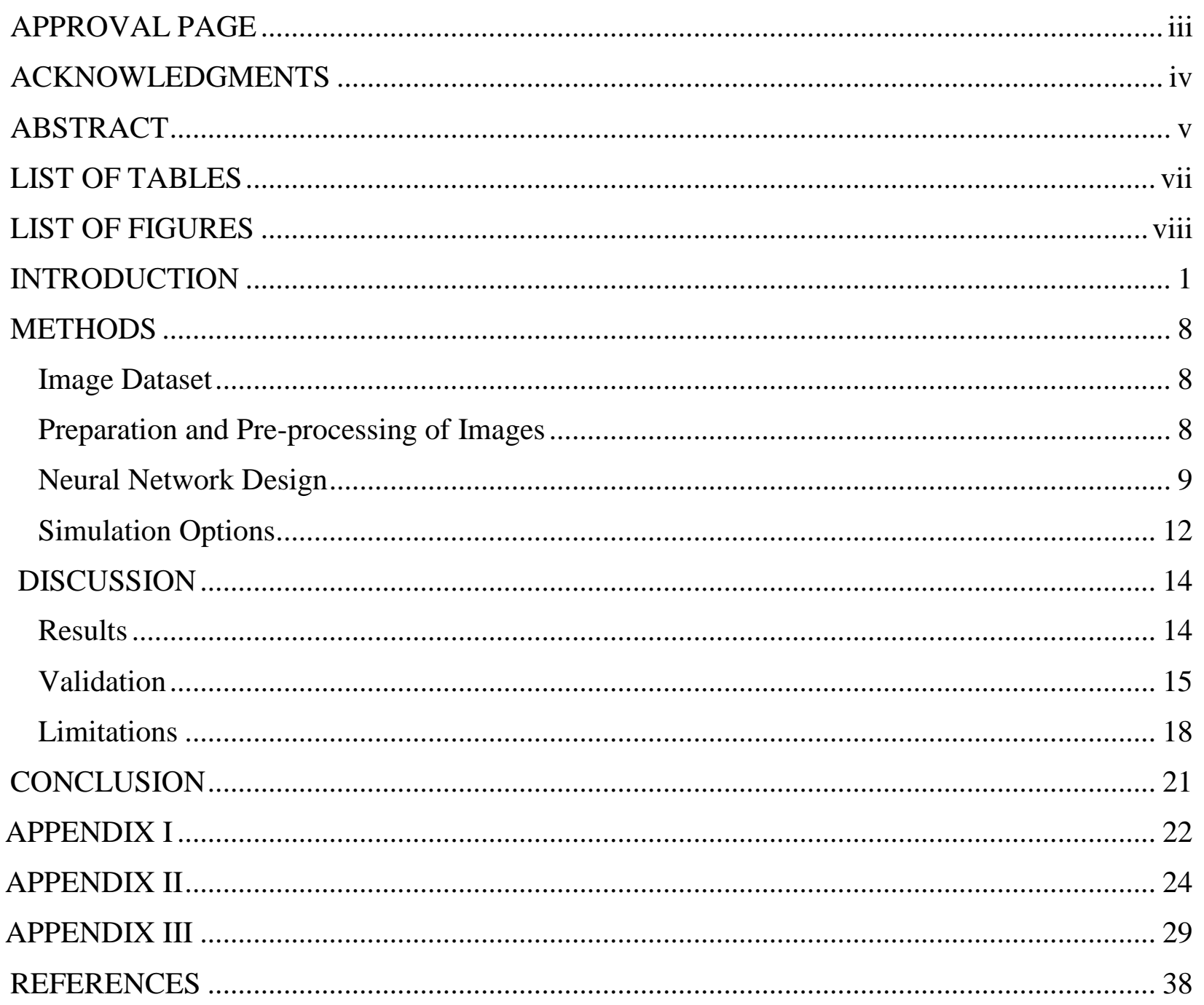




\section{LIST OF TABLES}

Table 1 - Average Classification accuracy of training and test images for 5 trials ..............15

Table 2 - Average Classification accuracy of normalized images for 5 trials ........................ 17 


\section{LIST OF FIGURES}

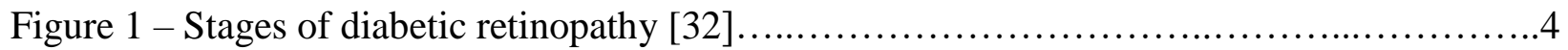
Figure 2 - Representations of various types of damage to capillaries in DR. (a) normal capillary.

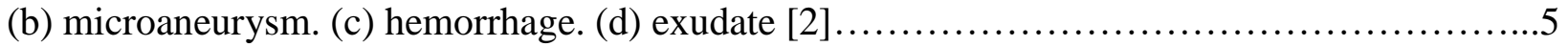

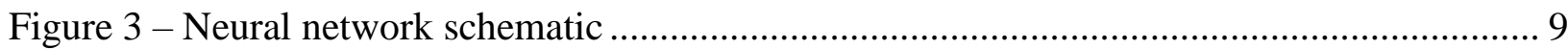

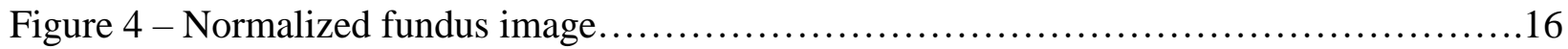

Figure 5 - Presence of eyelashes towards the bottom of the fundus image............................ 20

Figure 6 - Presence of camera artifacts (i.e. camera lens reflections) towards the top of the

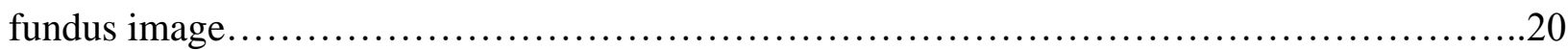




\section{INTRODUCTION}

Diabetic retinopathy (DR) is the leading cause of blindness among adults in the United States. It is the result of diabetes mellitus which itself is a global epidemic leading to a range of complications [1]. The main risk factors associated with diabetic retinopathy are duration of the diabetes, high blood sugar levels, and arterial hypertension [2][3][4]. At the onset, it affects the blood vessels in the retina, a photosensitive tissue found at the back of the eye. Overtime, under high elevation of glucose, the blood vessels can cause capillary endothelial damage which results in the cells along the capillary to become "leaky". The high glucose levels combined with endothelial damage lead to capillary occlusion which, in turn, leads to reduced blood flow [5]. If not treated, diabetic retinopathy progresses through four clinical stages (Figure 1) of mild non-proliferative, moderate non-proliferative, severe nonproliferative, which is sometimes subclassified into severe non-proliferative and very severe non-proliferative, and proliferative which is sometimes subclassified into nonhigh risk proliferative, and high risk proliferative, and advanced proliferative. In each stage there are problems that can occur (shown in Figure 2): such as microaneurysms (mild, moderate, severe, and proliferative stages), hemorrhages (moderate, severe, and proliferative stages), exudates (moderate, severe and proliferative stages), cotton-wool spots (severe, pre-proliferative, and proliferative stages), and intraretinal microvascular anomalies (proliferative stage) [5]. Microaneurysms are one of the earliest signs of diabetic retinopathy; classified as mild non-proliferative. They appear as red dots in the retina due to an expansion of the capillaries. In small numbers, microaneurysms do not affect the eyesight of a patient. Regardless, the number of 
microaneurysms can be used to estimate the regression or progression of diabetic retinopathy. Hemorrhages occur due to the rupture of small blood vessels in the retina, which results in red lesions. Hemorrhages occur deep in the retina where their shape is determined by their location or position. Even though hemorrhages are a cause of concern, they do not functionally disrupt the eye. Like microaneurysms, the number of hemorrhages can be used to estimate the progression or regression of diabetic retinopathy. Exudates define the yellow-white lesions that occur due to the leakage of plasma from capillaries near the macular region. Exudates away from the macular region have been observed but have not been found to cause serious problems. The first sighting of exudates means that diabetic retinopathy has progressed from the mild to the moderate non-proliferative stage. Cotton-wool spots are caused by failure of capillary circulation, which leads to the swelling of nerve fibers. They appear as pale or fuzzy areas on the retina. Solely, the cotton-wool spots do not cause visual problems, but their presence is graded as pre-proliferative or severe depending on how widespread they are. Intraretinal microvascular anomalies (IRMAs) or microvascular abnormalities represent the first sign of new blood vessels appearing. The cause of IRMAs is due to the dilation of capillaries and the shunts between the arteries and veins. Additionally, the retinal veins appear tortuous and irregular. Any patient with IRMAs is classified as proliferative, in which case they must seek medical help immediately.

It is estimated that about $30 \%$ to $50 \%$ of the diabetic population has retinopathy. In longitudinal studies, about 30\% of the affected individuals showed symptoms of diabetic retinopathy after 10 to 20 years of progression with diabetes 
mellitus. Of the $30 \%$ affected, $10 \%$ progressed to proliferative retinopathy, which puts patients at risk of blindness. In $90 \%$ of the cases examined, non-proliferative retinopathy is responsible for a serious loss of visual capacity. Non-proliferative retinopathy is also observed to occur more often in cases where diabetes onset started in adulthood, whereas proliferative retinopathy is present "generally", though not always in juvenile forms of the disease [5]. Another study, the classical Wisconsin Epidemiologic Study of Diabetic Retinopathy (WESDR), reported that about $75 \%$ of people diagnosed with diabetes mellitus showed signs and symptoms of diabetic retinopathy after 10 years. For those who developed early symptoms, about $66 \%$ of the people exhibited the severe stage of diabetic retinopathy. About $20 \%$ of those affected had the disease progress to proliferative diabetic retinopathy or diabetic macular odema, a subtype of diabetic retinopathy that involves the macula $[2][3][6][7]$.

Before 1961, to detect diabetic retinopathy, various diagnostic techniques were utilized. A visual acuity test was used to detect how well a person could see at various distances. A dilated eye exam was done to closely examine the retina and optic nerve for signs of damage. Additionally, a tonometry was done to detect abnormal pressure $(<12 \mathrm{mmHg}$ or $>22 \mathrm{mmHg}$ ) inside the eye [8]. With the advance of diagnostic medicine came the arrival of new imaging technologies. One such technology was fluorescein angiography. Fluorescein angiography involved using a fluorescent dye to mark the eye after which pictures were taken to identify vascular leakage and the presence of ischemia [9] [10]. Although traditionally used as the primary method in diagnosing diabetic retinopathy, fluorescein angiography was phased out over time in 


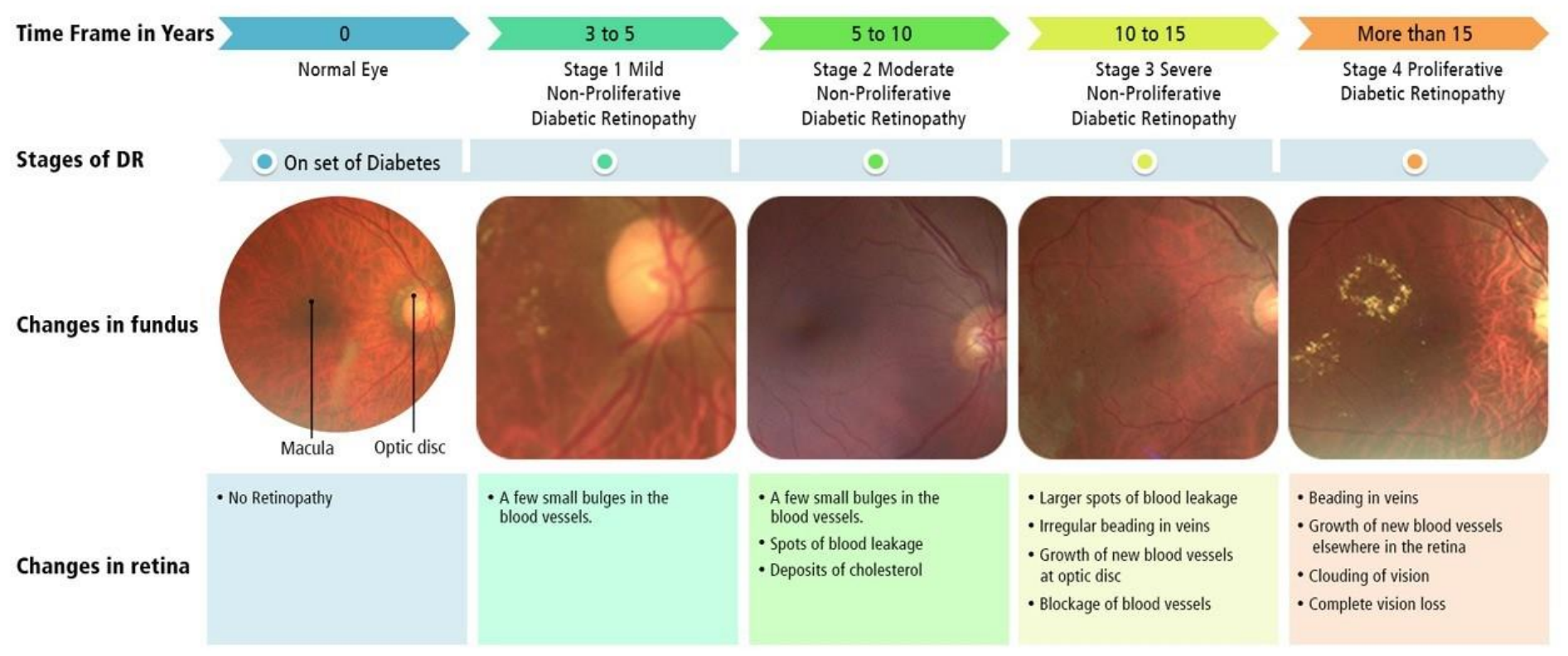

Figure 1-Stages of diabetic retinopathy [31].

preference of optical coherence tomography (OCT). Optical coherence tomography was considered a major technical innovation that did not require the need for pupil dilation and could be adopted with telemedicine to offer fast, accurate, systematic solutions in both developed and developing countries. [11] [12] [13]. As a noninvasive diagnostic technology, it creates high-resolution images of the retina to record 3D structural changes (e.g. odema) which otherwise cannot be deduced through a clinical examination of retinal pictures. At present, new fundus photography techniques are being tested and developed (e.g. wide-field fluorescein angiography) with some already introduced to the market, including the ultra-widefield (UWF) retinal imaging device, Optos 200 Tx, sold by Optos plc. Compared to current imaging techniques, ultra-widefield devices can image up to 200 degrees, thus providing more information to help clinicians accurately diagnose the condition. Additionally, these devices have a shorter image processing time and allow for image 
(a)
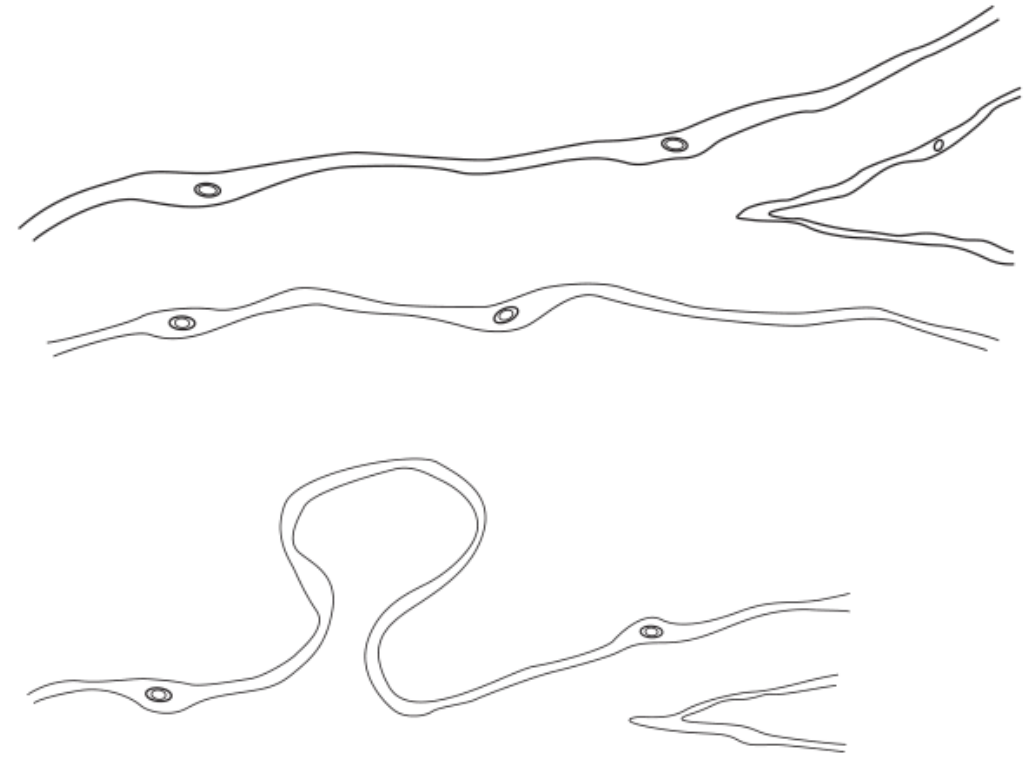

(b)

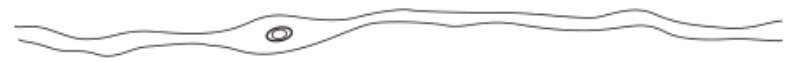

(c)

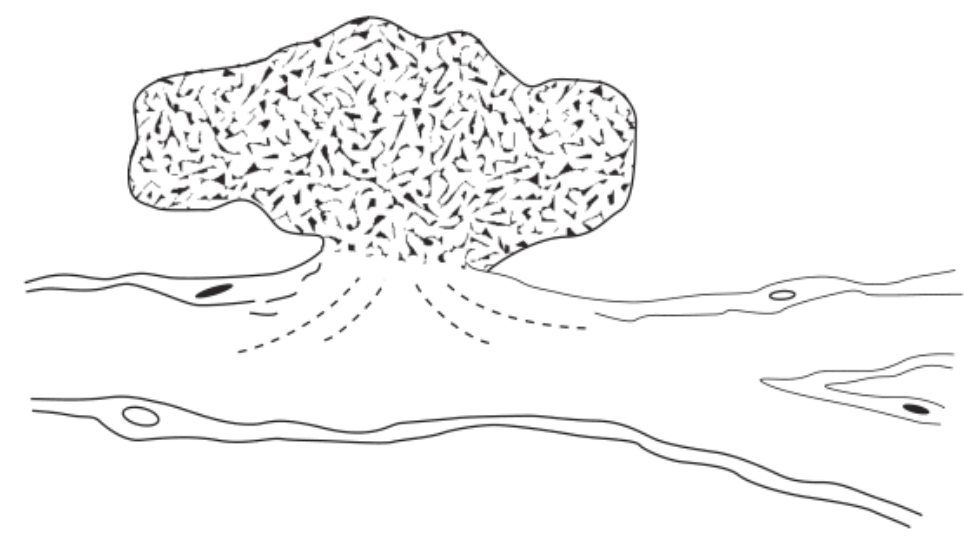

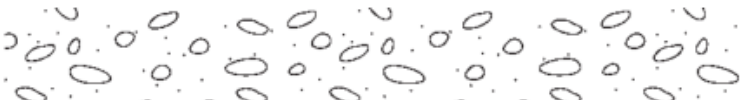

$$
\begin{aligned}
& 0.0 \text { o } 0 \text { o o o } 0.00
\end{aligned}
$$

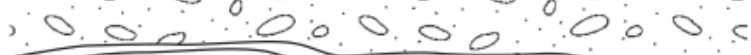

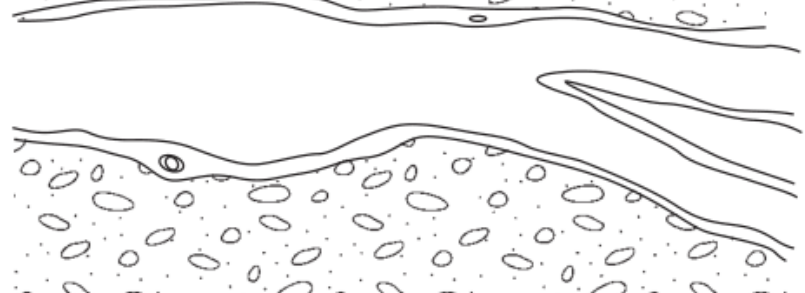

(d) 0 00 00000 0000

Figure 2 - Representations of various types of damage to capillaries in DR. (a) normal capillary. (b) microaneurysm. (c) hemorrhage. (d) exudate [5]. 
duplication and manipulation with ease [32].

With a recent rise in machine learning, applications in medical diagnostics have been explored due to their high classification accuracy. Deep learning, a subset of machine learning, uses multiple layers for feature extraction from an image. The input into each layer uses the output from the previous layer thus allowing every layer in the algorithm to independently learn the features. Deep learning models are typically trained using large datasets with an appropriate neural network to learn the features in an image without prior knowledge. The use of deep learning for medical images has been studied by LeCun et al. [14], Liu et al. [15], and Litjens et al. [16]. While most of the research on optical coherence tomography images has been explicitly based on segmenting features such as blood vessels and identifying the stage of diabetic retinopathy through a classifier, new research in the ophthalmologic field has been done by Pratt et al. [17], Sangeethaa et al. [18], and Chandrakumar [19] to detect diabetic retinopathy using the convoluted neural network (CNN). This network consists of three layers: input layer, hidden layer, and output layer. The hidden layer encompasses convolution and pooling layers to reduce computational time. As a part of deep learning, CNNs eliminate the need for manual feature extraction and can be retrained for new classification tasks. While the diagnostic accuracy of detecting diabetic retinopathy from OCT images has proven to be successful through CNN, the accuracy of ultra-widefield fundus images have yet to be fully investigated, especially involving various classes/stages. Therefore, the goals of this study are to develop and utilize CNN to help classify ultra-widefield fundus images into one of the 3 major classes of diabetic retinopathy; evaluate and compare 
the accuracy of the classification between different areas and sizes of fundus images; and explore the limitations of using CNN for ultra-widefield images. 


\section{METHODS}

\subsection{Image Dataset}

The images for this research were received from the Cleveland Clinic. There was a total of 62 images sent, 26 TIF format and 36 JPEG format, each of which represented 62 patients. The images were taken by a technician on an ultra-wide field ophthalmoscope with the resulting dimensions of most images being $3900 \times 3071$ (JPEG) and the remaining images being $3900 \times 3072(\mathrm{TIF})$. The horizontal and vertical resolution was 96 dpi (dots per inch). These images were then graded by an ophthalmologist and categorized into 1 of 7 classes based on the Early Treatment Diabetic Retinopathy Study (ETDRS) scale: mild (11 images), moderate (11 images), severe (15 images), very severe (14 images), non-high risk (4 images), high risk (3 images), and advanced (4 images).

\subsection{Preparation and Pre-processing of Images}

Due to the small number of images and uneven classes, the fundus images were organized into three main classes: mild (mild + moderate images), severe (severe + very severe images), and high risk (non-high risk + high risk + advanced images). This was done to balance the classes and provide enough training data for the machine learning algorithm. It is important to note that if there were "normal" images or images that showed no signs of diabetic retinopathy, then four classes would've been created to differentiate between normal, mild, severe, and high-risk diabetic retinopathy. Once the images were organized, using MATLAB, five masks at various diameters following a concentric pattern from the center of the fundus image were created. The masks were created to help isolate the region of interest (ROI) in the 
fundus images. After the creation of the mask, desired images with the ROI were cut out and converted to TIF format to prepare for training through the CNN. To begin pre-processing, the desired image dimension (e.g. $32 \times 32$ ) was chosen for the input layer. Once the images were inputted, they were shuffled to make MATLAB re-train with new images for each new training session. Then, image augmentation was utilized to randomly rotate and reflect the images to prevent overfitting and memorization of image features due to the small size of the image dataset.

\subsection{Neural Network Design}

A CNN was developed to classify fundus images into 3 classes. This network consists of a 2D convolution layer, batch normalization layer, rectified linear unit (ReLU) layer, max pooling layer, fully connected layer, softmax layer, and classification layer (Figure 3).
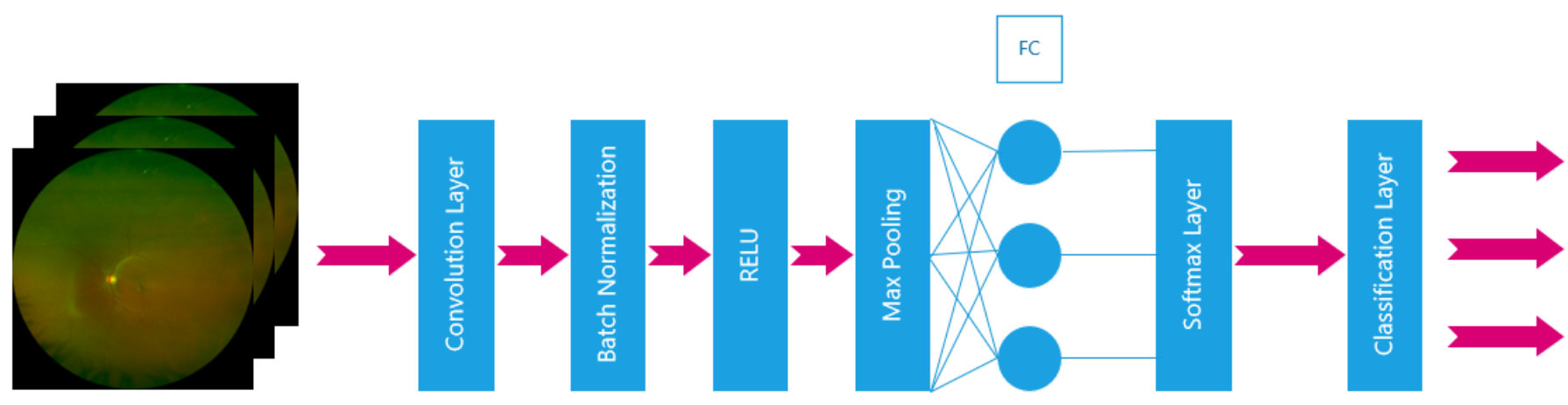

Figure 3 - Neural network schematic.

First, a 2D convolution layer was deployed, consisting of 5 filters with a stride (step size in which the filter moves) of 1 , which convolved the input layer by applying sliding filters horizontally and vertically. Given that the input pixel value of each fundus image examined is $x(a, b, c)$, the output of the convolutional layer is $y(w, y, z)$ - 
which represents the feature maps extracted by convolving $x(a, b, c)$ with $f(w, a, b, c)$ - is specified as follows:

$y(w, y, z)=\sum_{a=1}^{5} \sum_{b=1}^{5} \sum_{c=1}^{3} f(w, a, b, c) \cdot x(a+y-1, b+z-1, c)+b(w)$

$w$ represents the filter index where $w=1,2,3,4,5$. Similarly, $m=1,2,3,4 \ldots q$ and $n=$ $1,2,3,4 \ldots q$ where $q$ represents the input dimension of the image. The terms $b(w)$ and $f(w, a, b, c)$ represent the biases and weights of the filters, respectively [20]. Due to the small dataset and to avoid underfitting, the bias values and weights were kept at default values. The weights were initialized with the Glorot initializer, which is optimal for neural networks with a single hidden layer, with a small Gaussian value with zero mean and variance $=2 /($ fan-in + fan-out $)$ where fan-in $=$ filterSize $1 \times$ filterSize $2 \times$ Number of Input Channels and fan-out $=$ filterSize $1 \times$ filterSize $2 \times$ Number of Hidden Channels. The bias values were initialized with zeros since symmetry-breaking is provided through the weights. The number of neurons in a convolution layer is equivalent to the Map Size $\times$ Number of Filters. For example, if the input image is 32-by-32-by-3 the map size would be 15 -by- 15 since $(32-5+(1 \times 1)) / 2+1 \approx 15$. The Map Size is then multiplied by the Number of Filters resulting in the total number of neurons being $15 \times 15 \times 5=1125$.

After the output was obtained, a batch normalization layer was included after each convolution layer, normalizing the input and speeding up training while reducing the sensitivity to network initialization. As shown in equation (2), the layer functions by calculating the normalized activations by using the mean $\mu_{\mathrm{B}}$ and $\sigma_{\mathrm{B}}^{2}$ over the input channel and the mini batch. If the variance is very small, the $\varepsilon$ property comes into play by improving the numerical stability [21]. 


$$
\hat{x_{i}}=\frac{x_{i}-\mu_{B}}{\sqrt{\sigma_{B}^{2}+\epsilon}}
$$

The batch normalization layer was then followed by the rectified linear unit (ReLU) layer. This layer sets any value less than zero to zero; a type of threshold operation equivalent to equation (3) [22].

$$
f(x)= \begin{cases}x, & x \geq 0 \\ 0, & x<0\end{cases}
$$

A max pooling layer was implemented to perform down sampling by separating the input into "rectangular pooling regions and computing the maximum of each region"; specifically, a pooling layer with 2 filters and a stride of 2 [23]. Next, the fully connected layer was deployed to acquire sub-feature maps to help classify them into three classes. The outputSize was set to 3 which represented the three classes: mild, severe, and high risk. The fully connected layer functioned by multiplying the input by a weight matrix and then adding a bias to that matrix as shown in equation (4) [24] [25] [26]:

$$
f(t)=\sum_{k=1}^{4} \sum_{j=1}^{32} \sum_{i=1}^{32} \bar{w}(l, m, n) \cdot g(l, m, n)+\bar{b}(t)
$$

The terms $\bar{w}(l, m, n)$ and $\bar{b}(t)$ represent the weights and bias; $t$ is the index where $t=1$, 2, and 3. As mentioned before, no bias values or weights were specified to avoid underfitting. Following the fully connected layer was the softmax function, which calculates the probability distribution. The softmax function is also known as the normalized exponential function and is defined as such for multi-class classification problems: 


$$
\mathrm{P}\left(\mathrm{d}_{\mathrm{r}} \mid \mathrm{y}, \theta\right)=\frac{\mathrm{P}\left(\mathrm{y}, \theta \mid \mathrm{d}_{\mathrm{r}}\right) \mathrm{P}\left(\mathrm{d}_{\mathrm{r}}\right)}{\sum_{i=1}^{m} \mathrm{P}\left(\mathrm{y}, \theta \mid \mathrm{d}_{\mathrm{j}}\right) \mathrm{P}\left(\mathrm{d}_{\mathrm{j}}\right)}=\frac{e^{\left(b_{r}(y, \theta)\right)}}{\sum_{i=1}^{m} e^{\left(b_{j}(y, \theta)\right)}}
$$

The limits for the probability, $P\left(d_{r} \mid y, \theta\right)$, are from 0 to 1 and the term $\sum_{i=1}^{m} P\left(d_{j} \mid y, \theta\right)$ is equal to 1 . Additionally, $b_{r}=\ln \left(P\left(y, \theta \mid d_{r}\right) P\left(d_{r}\right)\right)$ where $P\left(y, \theta \mid d_{r}\right)$ is the conditional probability of the sample class $r$ and the prior probability $P\left(d_{r}\right)$ [27] . To successfully classify each fundus image, the cross-entropy loss was calculated in the final layer called the classification layer. The cross-entropy loss is defined as such:

$$
\text { Loss }=-\sum_{j=1}^{M} \sum_{i=1}^{H} s_{j i} \ln u_{j i}
$$

$H$ is the number of classes, $M$ is the number of samples, $s_{j i}$ is the indicator that correlates the $j$ th sample to the $i$ th class, and $u_{j i}$ is the output of the softmax function, associating the $j$ th input with the $i$ th class [27].

\subsection{Simulation Options}

Once the neural network was designed, the simulation options were specified. The learn rate of 0.01 was specified along with the maximum number of epochs of 100 and a mini-batch size of 48. For network training, 52 randomly selected images were used. The remaining images (10) were used for classification. The command shuffle every-epoch was used to shuffle the training data before each epoch and also to take advantage of the batch normalization effect, thus making the training of the 
neural network an effortless optimization problem. The algorithm used for training is the stochastic gradient descent with momentum (SGDM), which is defined as

$$
\theta_{n+1}=\theta n-\beta \nabla F\left(\theta_{n}\right)+\eta\left(\theta_{n}-\theta_{n-1}\right)
$$

where $\theta$ is the parameter vector $n$ is the iteration number, $\beta$ is the learn rate $(\beta>0)$, $\eta\left(\theta_{n}-\theta_{n-1}\right)$ is the momentum term/parameter, and $F(\theta)$ is the loss function. This SGDM algorithm minimizes the cross-entropy loss across the mini-batch by proceeding incrementally at each iteration in the direction of the gradient loss. Adding the momentum term, value of 0.9 , helps reduce oscillations along the path of steep descent toward the optimum value [28]. 


\section{DISCUSSION}

\subsection{Results}

The neural network was simulated for a total of 25 times with 5 trials for each image size: $32 \times 32,64 \times 64,128 \times 128,256 \times 256$, and $512 \times 512$. The average simulation/processing time ranged from 25 seconds for $32 \times 32$ images, to 22 minutes for $512 \times 512$ images. A confusion matrix was created for each of the image sizes to obtain statistical measures of performance, such as sensitivity, false negative rate, precision, and the false discovery rate. The sensitivity was $70 \%$ for $32 \times 32$ images, $65 \%$ for $64 \times 64$ images, $60 \%$ for $128 \times 128$ images, $50 \%$ for $256 \times 256$ images and $50 \%$ for $512 \times 512$ images. The sensitivity decreased as the size of the images increased; the opposite is true for the false negative rate. Similarly, the precision was $65 \%$ for $32 \times 32$ images, $65 \%$ for $64 \times 64$ images, $60 \%$ for $128 \times 128$ images, $50 \%$ for $256 \times 256$ images, and $50 \%$ for $512 \times 512$ images. The precision decreased as the size of the images increased, while the false discovery rate increased as the size of the images increased (Appendix II). These statistical measures suggest that a combination of a small image dataset and uneven classes may contribute to an increase in type I and type II errors thus leading to irrelevant results. The classification accuracy of the training and the test sets was determined by adding the number of correctly classified images divided by the total number of classified images; 52 images total for training and 10 images total for testing. As shown in Table 1, the training accuracy for the $32 \times 32,64 \times 64$, and $128 \times 128$ images ranged from $92 \%$ to $97 \%$. For both the $256 \times 256$ and $512 \times 512$ images, the training accuracy was $46 \%$. The training accuracy represents the accuracy of the neural network model on the image sets on which it was constructed. While within 100 epochs, most of the images up to size of 
$128 \times 128$ approached $>90 \%$ training accuracy, the images of sizes $256 \times 256$ and $512 \times 512$ had their respective training accuracy plateau at $46 \%$ with the 45 th iteration (or epoch).

Table 1 - Average classification accuracy of training and test images for 5 trials

\begin{tabular}{|l|r|r|}
\hline Image Sizes & Avg. Training Accuracy (\%) & Avg. Test Accuracy (\%) \\
\hline $32 \times 32$ & 92.692 & 50.000 \\
\hline $64 \times 64$ & 97.307 & 48.000 \\
\hline $128 \times 128$ & 96.923 & 52.000 \\
\hline $256 \times 256$ & 46.153 & 50.000 \\
\hline $512 \times 512$ & 46.538 & 50.000 \\
\hline
\end{tabular}

The plateauing of the accuracy is significant because it means that the algorithm has hit convergence, where further training is likely to degrade the test accuracy and the training accuracy and result in overfitting. The test accuracy is the accuracy of the images the model has yet to see. Although the test accuracy of all the images was around 50\%, an important observation to note is that the test accuracy did not improve as the image size increased, as one would intuitively believe since larger images have more pixel data. This shows, as is commonly known in the ophthalmologic field, that the majority ( 90\%) of cases, the information for proper diagnosis is around the optic nerve and macula region of the eye. Moreover, this suggests that different ROIs do not impact the diagnosis of DR.

\subsection{Validation}

While the accuracy results were not ideal, they provide some insight into why they are low. Noticing the discrepancy between training accuracy and test accuracy, one would think that overfitting might be the reason. So, to test this hypothesis and to verify 
the results, two methods were used: image normalization and transfer learning.

Image normalization is the process of changing the histogram values of an image to bring it to a range of intensity values that is "normal" (normal distribution). This avoids the influence of very low and very high noise in the image. In machine learning, image normalization is sometimes used to make learning more stable and to make the neural network generalize favorably to unseen data by reducing the variability between the training set and the testing set [18]. For this simulation, all images were normalized by taking each individual color channel and dividing them by the magnitude of all three (RGB) color channels (Figure 4).

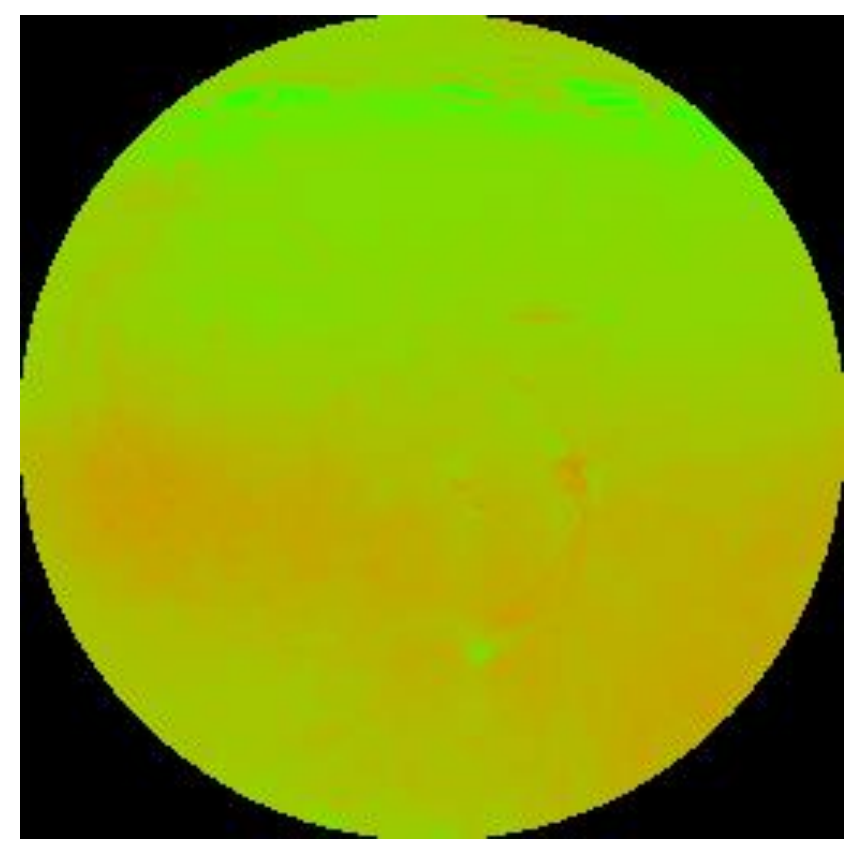

Figure 4-Normalized fundus image.

After normalization, the images were trained and tested using the $\mathrm{CNN}$ algorithm and produced the following results (Table 2): 
Table 2 - Average classification accuracy of normalized images for 5 trials

\begin{tabular}{|l|r|r|}
\hline Image Sizes & Avg. Training Accuracy (\%) & Avg. Test Accuracy (\%) \\
\hline $32 \times 32$ & 88.461 & 50.000 \\
\hline $64 \times 64$ & 96.153 & 50.000 \\
\hline $128 \times 128$ & 94.423 & 50.000 \\
\hline $256 \times 256$ & 46.153 & 50.000 \\
\hline $512 \times 512$ & 46.153 & 50.000 \\
\hline
\end{tabular}

The results show a similar pattern to the original results where the training accuracy is relatively high for image sizes of $32 \times 32,64 \times 64$, and $128 \times 128$ followed by a steep decrease to $46 \%$ for image sizes of $256 \times 256$ and $512 \times 512$. The test accuracy did not show a significant difference from the original results, staying around 50\%. Two things that can be concluded from this method is that overfitting is not a cause for the discrepancy in the accuracy values and that the varied levels of lighting in the original images, which could possibly affect pixel intensity values and create unnecessary variation, did not impact the test accuracy.

Transfer learning utilizes a model that has been trained on a larger dataset which can be fine-tuned for a new task or new dataset; especially small datasets $(<1000$ images). Compared to the performance of a model that is trained from scratch, it achieves higher network performance in a short amount of time. The pre-trained model used for transfer learning for this study is called AlexNet. This model was developed by Alex Krizhevsky utilizing the $\mathrm{CNN}$ algorithm. It has been trained on 1.2 million images from the ImageNet Dataset (http://image-net.org/index) with image dimensions of $227 \times 227$. The structure of the model consists of 5 convolution layers which together with the 
remaining layers add to a total of 23 [29]. Once the model is selected, the last classification layer is replaced for a new classification task. Since an image size of $227 \times 227$ is required as the input, fundus images with dimensions of $256 \times 256$ and $512 \times 512$ were used by utilizing the function augmentedImageDatastore to automatically resize the images with no loss of data. Then, the model was simulated, and the training accuracy and test accuracy were obtained. Similar to the results of the normalized images and the original results, after 5 trials the average test accuracy was found to be $50 \%$. On the other hand, the training accuracy was $100 \%$. The high training accuracy is most likely due to overfitting since the transfer learning model did not include an image augmentation step to account for overfitting. While the use of transfer learning to improve classification of DR has been done using AlexNet [30], in this case transfer learning showed no significant improvement compared to the original method that was done from scratch. This strongly suggests that any additional convolution layers are unnecessary since the algorithm has already extracted all the features with one convolution layer for successful classification.

\subsection{Limitations}

The reason that neither the original method nor the validation methods were able to achieve greater than $50 \%$ accuracy can be attributed to one of many reasons which are also the limitations of this study. One limitation, perhaps the most impactful limitation, was the small amount of data available. While researchers have been able to use a small dataset to detect DR from 140 OCT images with a classification accuracy of 93\%, no current literature exists demonstrating this with ultra-widefield fundus images. One could argue that with a more complex algorithm, i.e. with additional convolution layers, one 
could achieve better performance [18]. However, as stated previously that even with additional layers, the algorithm showed no improvement in performance.

Another limitation was the unbalanced image classes caused by a lack of diseased images. Initially, the images were rearranged into four classes where the classification accuracy (test accuracy) was found to be $35 \%$. With the images rearranged into 2 classes, the classification accuracy was $60 \%$ (see Appendix III for probability values). Although decreasing the number of classes does increase accuracy, it is not beneficial for doctors since they would need to further examine the fundus images to determine the best treatment plan. Other ways to tackle the unbalanced image classes were to balance the classes by removing images from larger classes or by adjusting weights and bias components in the algorithm. By having an equal number of images in each of the three classes, the training accuracy decreased to around $80 \%$ for most of the image sizes and the test accuracy decreased for three of the image sizes ranging from $28 \%$ to $39 \%$ (Appendix I). There were additional trials done by adjusting the weights to "zeros" and changing the bias to "ones". The average classification accuracy for all images was about $23 \%$ thus severely underfitting and resulting in high training loss; neither able to model the training images nor able to generalize the test images.

A third limitation in this study was the presence of unnecessary features such as eyelashes (Figure 5) and camera artifacts (Figure 6) in some of the images. The presence of eyelashes caused the $\mathrm{CNN}$ algorithm to learn the unnecessary features and thus deteriorate the classification accuracy, especially for larger image sizes $(256 \times 256$ and $512 \times 512)$. 


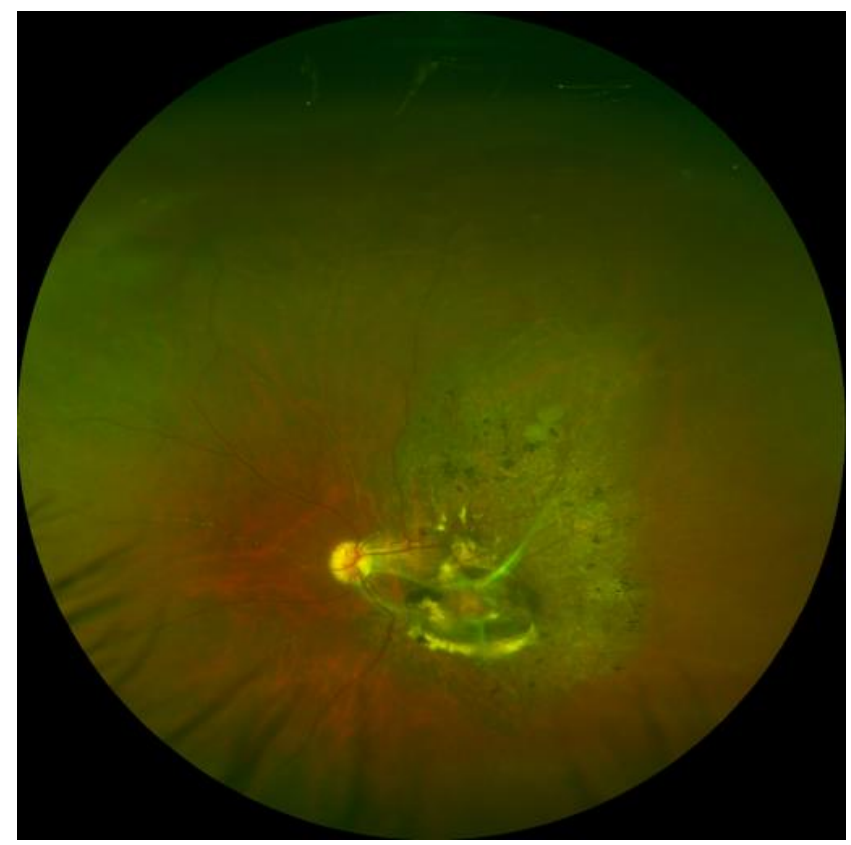

Figure 5-Presence of eyelashes towards the bottom of the fundus image.

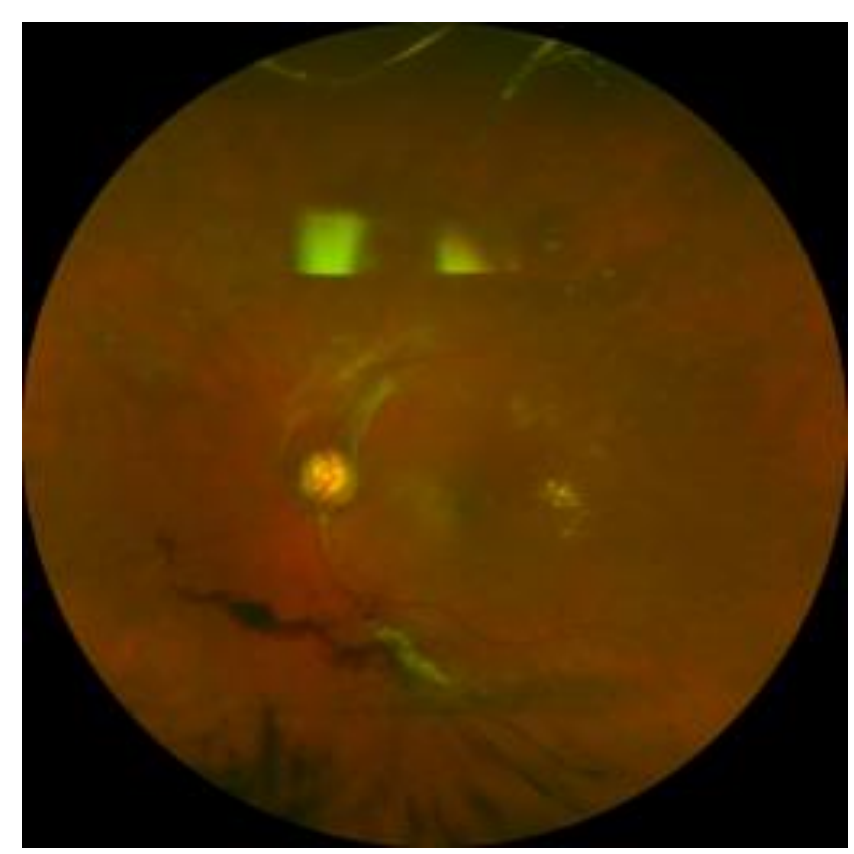

Figure 6-Presence of camera artifacts (i.e. camera lens reflection) towards the top of the fundus image 


\section{CONCLUSION}

The goals of this study were to develop an algorithm to classify ultra-widefield fundus images into one of the 3 major classes of diabetic retinopathy, evaluate and compare the accuracy of the classification between different areas and sizes of fundus images, and explore the limitations of using $\mathrm{CNN}$ for ultra-widefield images. Validation methods such as image normalization and transfer learning were also done to calculate the classification accuracy and justify the algorithm that was developed from scratch. While the results are not ideal, they demonstrate the potential for applying deep learning algorithms to ultra-widefield images and the need for doctors to further examine the diagnosis to account for false positives and/or misdiagnosis.

Future work in this subject would include improvements to the CNN algorithm as more images are obtained in order to improve the classification accuracy. Additional research would be done to determine if the file type of an image (e.g. JPEG or TIF) impacts the classification accuracy. Also, instead of concentric circles which mainly focus on the optic nerve, different sections of an image may be isolated/cut out where the accuracy of different regions of the eye may be compared to see if the classification accuracy solely relies on the optic nerve. Lastly, some pre-processing would be done to remove artifacts such as eyelashes from the images to reduce the number of features to be learned by the $\mathrm{CNN}$ algorithm; only if the flexibility is available to do so i.e. large amount of data available where removal of some images will not drastically impact the classification accuracy. 
APPENDIX I - Classification Data

\section{A. Balanced classes}

\begin{tabular}{|c|c|c|c|}
\hline Trials & $\begin{array}{l}\text { Image } \\
\text { size }\end{array}$ & $\begin{array}{l}\text { Training } \\
\text { Accuracy }\end{array}$ & Test Accuracy \\
\hline 1 & \multirow{5}{*}{$32 * 32$} & 0.5439 & 0.4000 \\
\hline 2 & & 0.6667 & 0.6000 \\
\hline 3 & & 0.5995 & 0.6000 \\
\hline 4 & & 0.5789 & 0.6000 \\
\hline 5 & & 0.6140 & 0.4000 \\
\hline & Avg. (\%) & 60.06 & 52.000 \\
\hline 1 & \multirow{5}{*}{$64 * 64$} & 0.8421 & 0.4000 \\
\hline 2 & & 0.6140 & 0.4000 \\
\hline 3 & & 0.7719 & 0.2000 \\
\hline 4 & & 0.6140 & 0.4000 \\
\hline \multirow[t]{2}{*}{5} & & 0.8421 & 0.4000 \\
\hline & Avg. (\%) & 73.682 & 36.000 \\
\hline 1 & \multirow{5}{*}{$128 * 128$} & 1 & 0.2759 \\
\hline 2 & & 0.7018 & 0.2000 \\
\hline 3 & & 0.7719 & 0.6000 \\
\hline 4 & & 0.7576 & 0.4000 \\
\hline \multirow[t]{2}{*}{5} & & 0.9394 & 0.4828 \\
\hline & Avg. (\%) & 83.414 & 39.174 \\
\hline 1 & \multirow{5}{*}{$256 * 256$} & 0.8485 & 0.3103 \\
\hline 2 & & 0.6061 & 0.3793 \\
\hline 3 & & 0.9394 & 0.1724 \\
\hline 4 & & 0.6970 & 0.2069 \\
\hline \multirow[t]{2}{*}{5} & & 0.9394 & 0.3448 \\
\hline & Avg. (\%) & 80.608 & 28.274 \\
\hline 1 & \multirow{5}{*}{$512 * 512$} & 0.8788 & 0.2414 \\
\hline 2 & & 0.9091 & 0.3103 \\
\hline 3 & & 0.8596 & 0.6000 \\
\hline 4 & & 0.6667 & 0.8000 \\
\hline \multirow[t]{2}{*}{5} & & 0.8741 & 0.6333 \\
\hline & Avg. (\%) & 83.766 & 51.7 \\
\hline
\end{tabular}




\section{B. Unbalanced classes}

\begin{tabular}{|c|c|c|c|}
\hline Trials & $\begin{array}{l}\text { Image } \\
\text { size }\end{array}$ & $\begin{array}{l}\text { Training } \\
\text { Accuracy }\end{array}$ & $\begin{array}{l}\text { Test } \\
\text { Accuracy }\end{array}$ \\
\hline 1 & \multirow{5}{*}{$32 * 32$} & 0.9038 & 0.6000 \\
\hline 2 & & 0.9615 & 0.7000 \\
\hline 3 & & 0.9038 & 0.4000 \\
\hline 4 & & 0.9423 & 0.3000 \\
\hline \multirow[t]{2}{*}{5} & & 0.9230 & 0.5000 \\
\hline & Avg. (\%) & 92.692 & 50.000 \\
\hline 1 & \multirow{5}{*}{$64 * 64$} & 1.0000 & 0.4000 \\
\hline 2 & & 0.9807 & 0.5000 \\
\hline 3 & & 0.9807 & 0.6000 \\
\hline 4 & & 0.9615 & 0.5000 \\
\hline \multirow[t]{2}{*}{5} & & 0.9423 & 0.4000 \\
\hline & Avg. (\%) & 97.308 & 48.000 \\
\hline 1 & \multirow{5}{*}{$128 * 128$} & 0.9038 & 0.6000 \\
\hline 2 & & 0.9615 & 0.5000 \\
\hline 3 & & 1.0000 & 0.4000 \\
\hline 4 & & 0.9807 & 0.5000 \\
\hline \multirow[t]{2}{*}{5} & & 1.0000 & 0.6000 \\
\hline & Avg. (\%) & 96.923 & 52.000 \\
\hline 1 & \multirow{5}{*}{$256 * 256$} & 0.4615 & 0.5000 \\
\hline 2 & & 0.4615 & 0.5000 \\
\hline 3 & & 0.4615 & 0.5000 \\
\hline 4 & & 0.4615 & 0.5000 \\
\hline \multirow[t]{2}{*}{5} & & 0.4615 & 0.5000 \\
\hline & Avg. (\%) & 46.153 & 50.000 \\
\hline 1 & \multirow{5}{*}{$512 * 512$} & 0.4807 & 0.5000 \\
\hline 2 & & 0.4615 & 0.5000 \\
\hline 3 & & 0.4615 & 0.5000 \\
\hline 4 & & 0.4615 & 0.5000 \\
\hline \multirow[t]{2}{*}{5} & & 0.4615 & 0.5000 \\
\hline & Avg. (\%) & 46.538 & 50.000 \\
\hline
\end{tabular}




\section{APPENDIX II - Confusion Matrices}

Far right column $=$ precision, false discovery rate

Bottom row $=$ sensitivity, miss rate

Bottom right corner $=$ overall accuracy of current trial, overall inaccuracy of current trial

\section{$32 \times 32$}

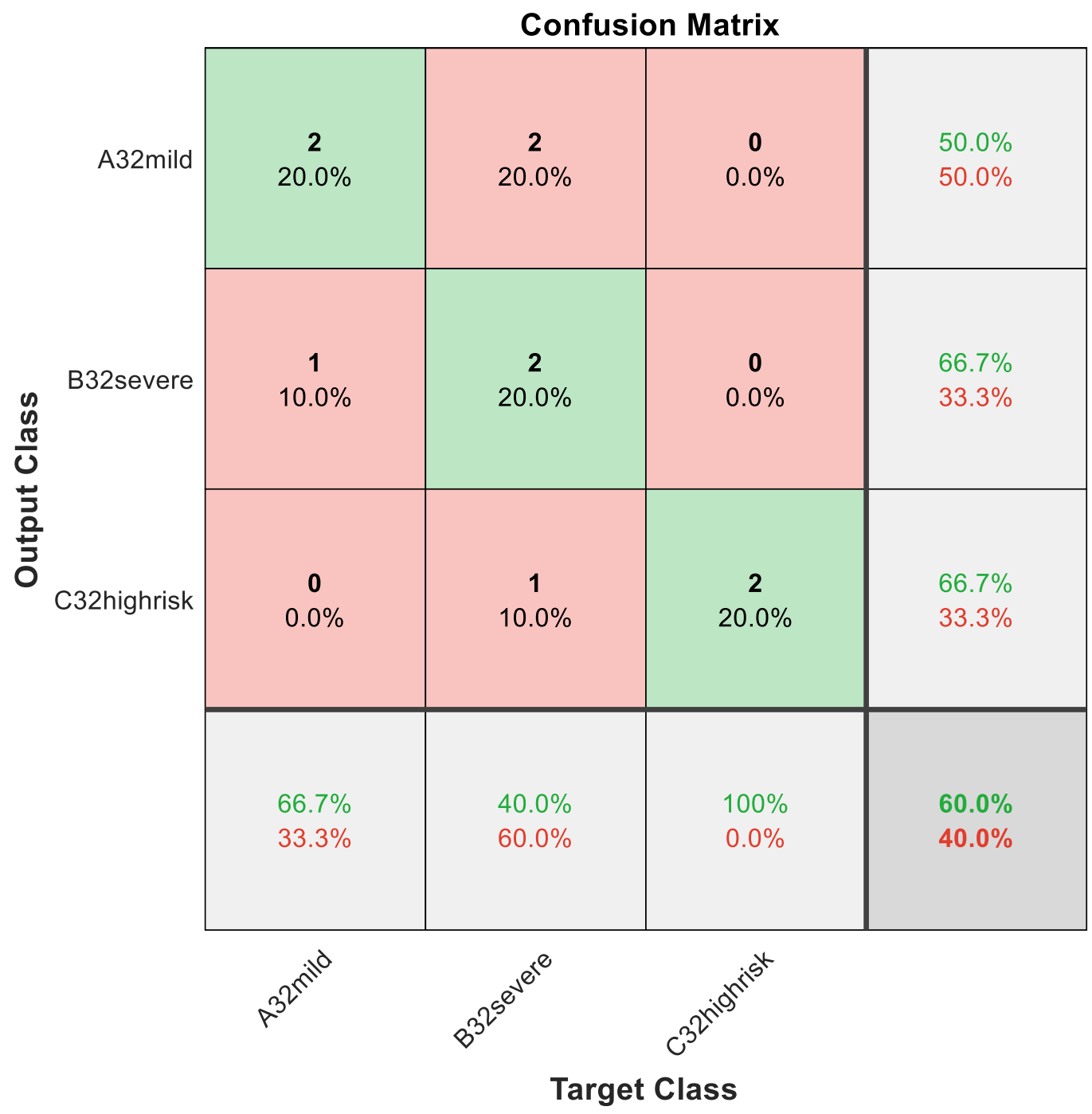




\section{$64 \times 64$}

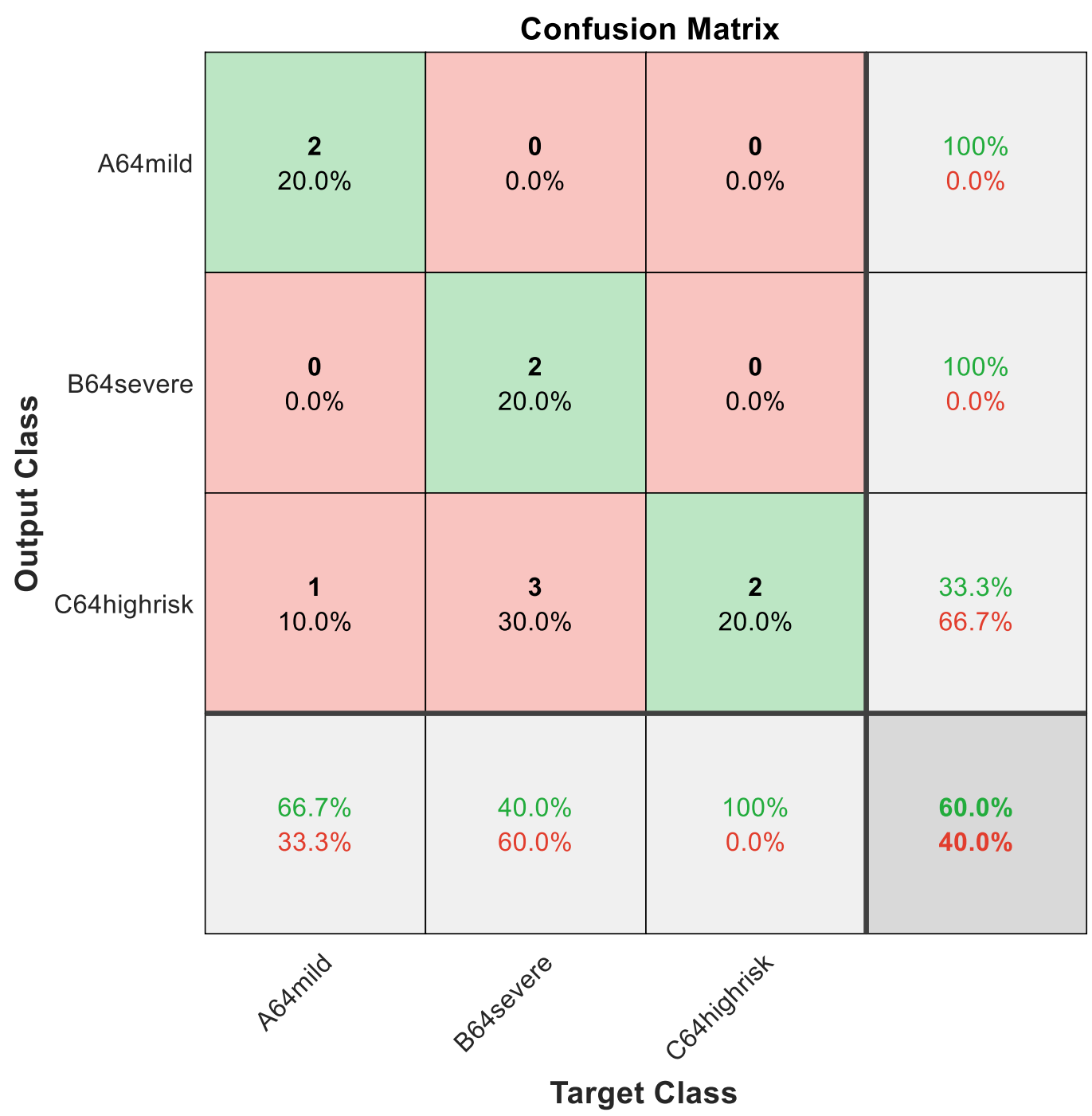




\section{$128 \times 128$}

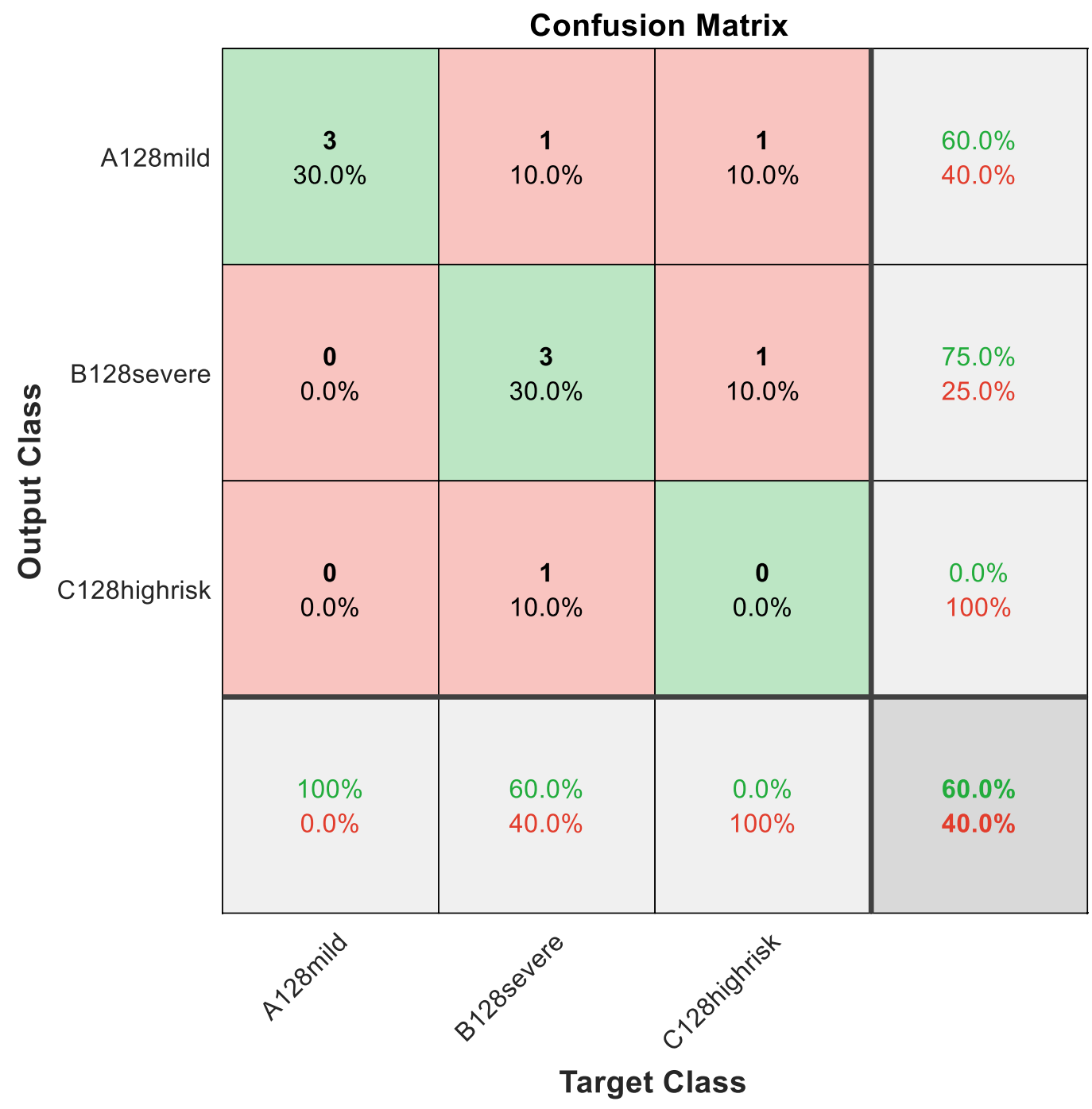




\section{$256 \times 256$}

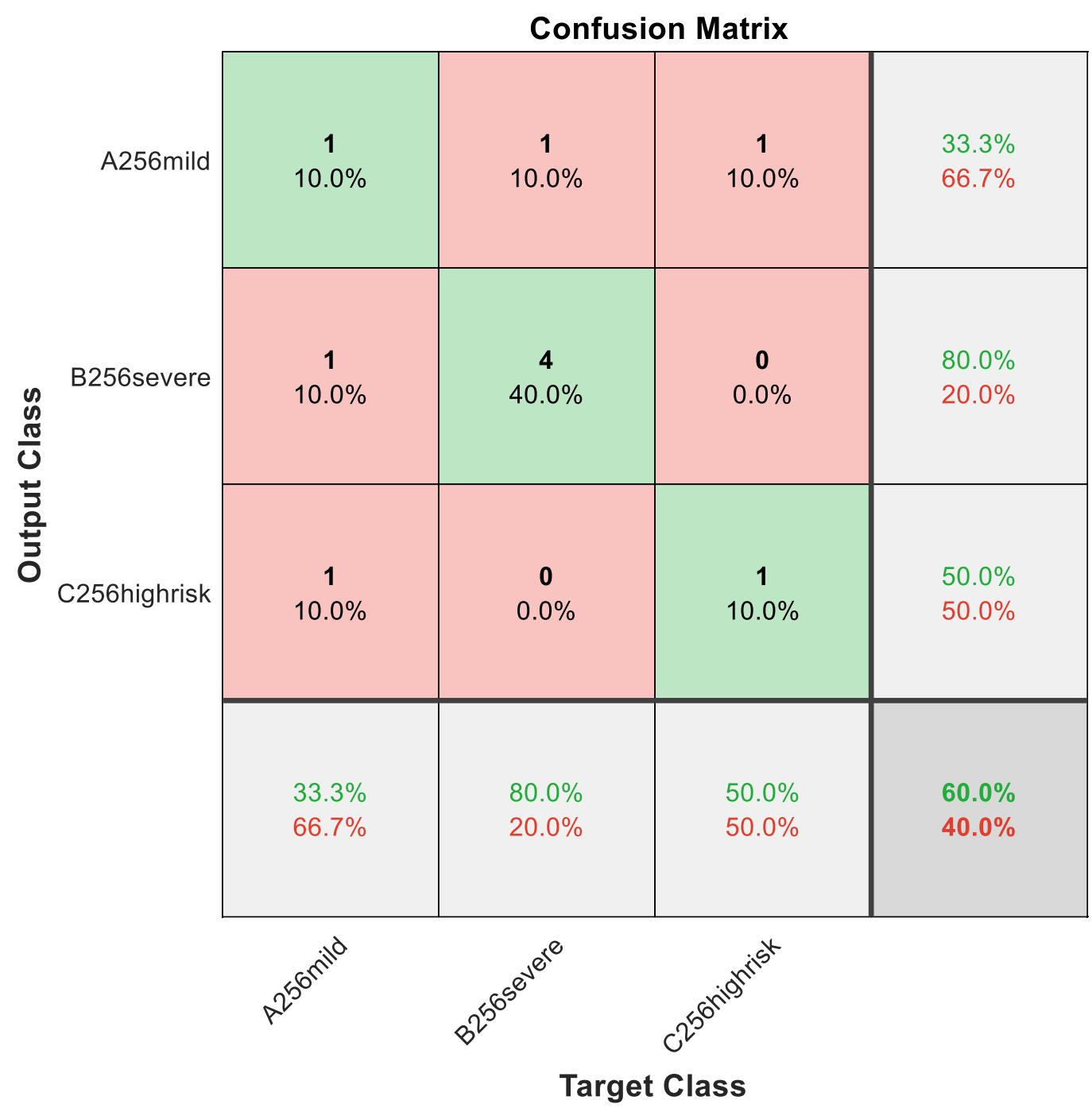




\section{$512 \times 512$}

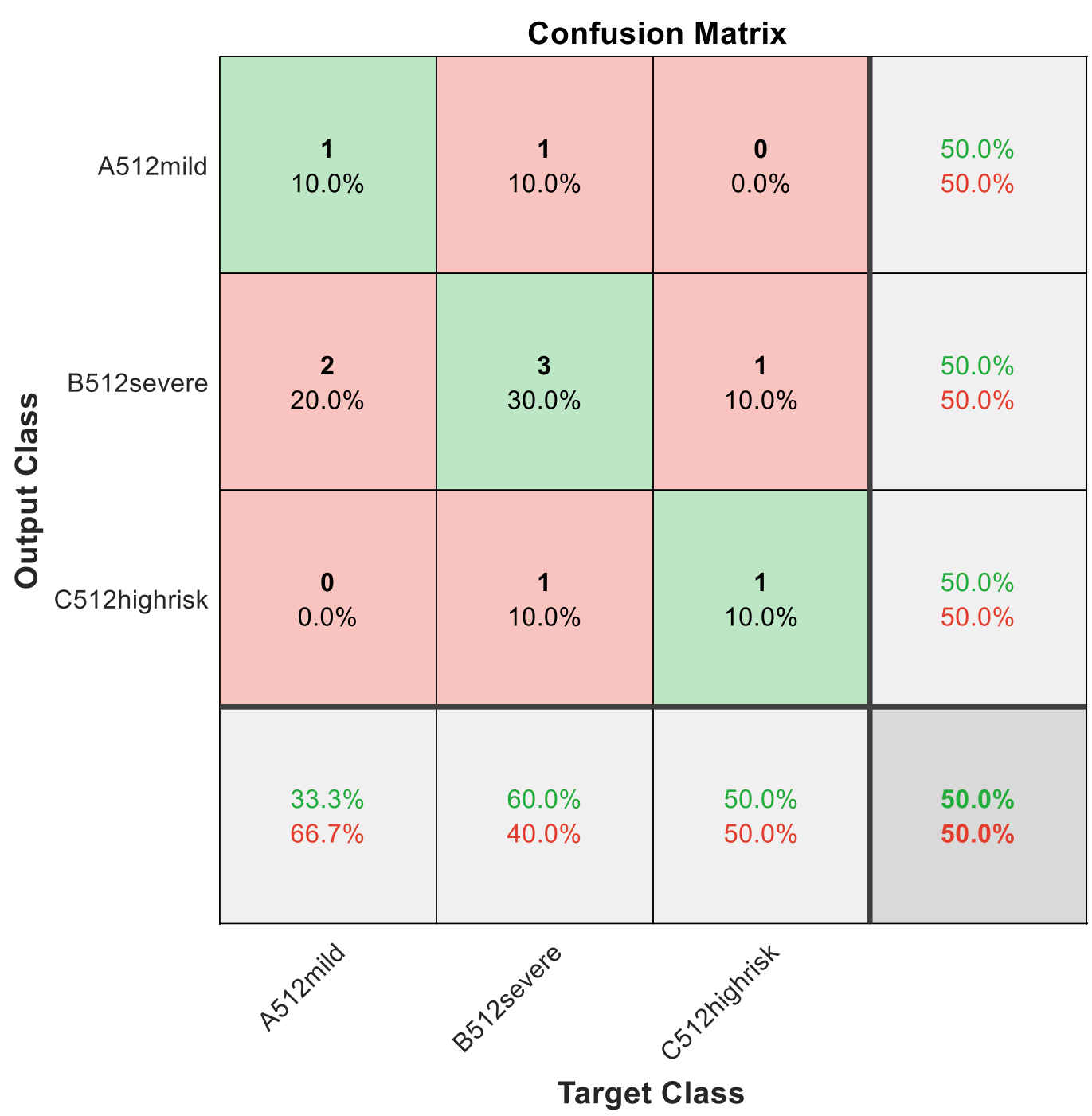




\section{APPENDIX III - Probability Data}

Red $=$ incorrectly predicted

\section{$32 \times 32$}

\begin{tabular}{|c|c|c|c|}
\hline Patient & Type & $\begin{array}{l}\text { P (Abnormal) } \\
{[32 * 32]}\end{array}$ & $\begin{array}{l}\mathrm{P}(\text { Normal) } \\
{[32 * 32]}\end{array}$ \\
\hline 10001418 OS & abnormal & 0.99998534 & $1.47 \mathrm{E}-05$ \\
\hline 10022953 OS & abnormal & $2.43 \mathrm{E}-07$ & 0.99999976 \\
\hline 10029705-20180416@112408-L4 & abnormal & 0.99998879 & $1.12 \mathrm{E}-05$ \\
\hline 10029705-20180416@112408-R3 & abnormal & 0.13589142 & 0.86410856 \\
\hline 10041101 OD & abnormal & 0.005335684 & 0.99466431 \\
\hline 10041101 OS & abnormal & $3.72 \mathrm{E}-08$ & 1 \\
\hline 10046318 OS & abnormal & 0.14558524 & 0.85441476 \\
\hline 10055620 OD & abnormal & 0.99221849 & 0.007781468 \\
\hline 10055620 OS & abnormal & 0.00250658 & 0.99749339 \\
\hline 10065949 OS & abnormal & 0.0087861 & 0.99121398 \\
\hline 10068165 OD & abnormal & 0.040095784 & 0.95990425 \\
\hline 10075046 OD & abnormal & 0.80176336 & 0.19823663 \\
\hline 10075046 OS & abnormal & 0.80258894 & 0.19741113 \\
\hline 10077406 OD & abnormal & 0.66958404 & 0.33041599 \\
\hline 10077406 OS & abnormal & 0.99926466 & 0.000735337 \\
\hline 10084968 OS & abnormal & 0.16454743 & 0.83545256 \\
\hline 10086708 OS & abnormal & $6.59 \mathrm{E}-10$ & 1 \\
\hline 10117924-20180220@102014-L4 & abnormal & 0.79679787 & 0.20320213 \\
\hline 10145754-20180415@105849-L2 & abnormal & 0.99968874 & 0.000311262 \\
\hline 10145754-20180415@105849-R1 & abnormal & 0.99999964 & $3.16 \mathrm{E}-07$ \\
\hline 10148705-20180425@100700-R3 & abnormal & 0.9999944 & $5.65 \mathrm{E}-06$ \\
\hline 10149811-20180225@090046-R1 & abnormal & 0.99999905 & $9.38 \mathrm{E}-07$ \\
\hline 10158969-20180506@090506-R1 & abnormal & 0.99999976 & $2.90 \mathrm{E}-07$ \\
\hline 10001091 OD & abnormal & 0.38385868 & 0.61614132 \\
\hline 10001091 OS & abnormal & 0.23049685 & 0.76950318 \\
\hline 10000464 OS & mild/moderate & $2.74 \mathrm{E}-09$ & 1 \\
\hline 10000484 OD & mild/moderate & 0.9986406 & 0.001359399 \\
\hline 10001418 OD & mild/moderate & 0.99864513 & 0.001354832 \\
\hline 10004898 OS & mild/moderate & 4.69E-15 & 1 \\
\hline 10008285 OS & mild/moderate & 0.73723733 & 0.26276267 \\
\hline 10013404 OD & mild/moderate & 0.96721429 & 0.032785717 \\
\hline 10018169 OD & mild/moderate & 0.96985573 & 0.03014431 \\
\hline 10022953 OD & mild/moderate & 0.026244124 & 0.9737559 \\
\hline 10022979-20180213@155058-R1 & mild/moderate & 0.99999571 & $4.26 \mathrm{E}-06$ \\
\hline 10025329 OD & mild/moderate & 0.99999142 & $8.55 \mathrm{E}-06$ \\
\hline
\end{tabular}




\begin{tabular}{|c|c|c|c|}
\hline 10028832 OD & mild/moderate & 0.99961448 & 0.000385448 \\
\hline 10028832 OS & mild/moderate & $3.78 \mathrm{E}-06$ & 0.99999619 \\
\hline 10037058 OD & mild/moderate & 0.44739011 & 0.55260986 \\
\hline 10037058 OS & mild/moderate & 0.1597831 & 0.84021693 \\
\hline 10041971 OD & mild/moderate & 0.048188951 & 0.95181108 \\
\hline 10041971 OS & mild/moderate & $5.42 \mathrm{E}-10$ & 1 \\
\hline 10047851 & mild/moderate & 0.5488714 & 0.45112863 \\
\hline 10047851 OD & mild/moderate & 0.99706465 & 0.002935403 \\
\hline 10051334 OD & mild/moderate & 8.39E-06 & 0.99999166 \\
\hline 10051334 OS & mild/moderate & 0.018040443 & 0.98195964 \\
\hline 10059609-20180325@094033-L3 & mild/moderate & 0.090828925 & 0.9091711 \\
\hline 10065949 OD & mild/moderate & 0.99982738 & 0.000172549 \\
\hline 10068165 OS & mild/moderate & $1.01 \mathrm{E}-05$ & 0.99998987 \\
\hline 10072783-20180121@092503-L2 & mild/moderate & 0.97144598 & 0.028554037 \\
\hline 10075925 OD & mild/moderate & 0.99999118 & $8.78 \mathrm{E}-06$ \\
\hline 10081192 OS & mild/moderate & $8.48 \mathrm{E}-06$ & 0.99999154 \\
\hline 10084968 OD & mild/moderate & 0.01174185 & 0.98825818 \\
\hline 10086708 OD & mild/moderate & 0.000257731 & 0.99974221 \\
\hline 10088791 OD & mild/moderate & 1 & $2.72 \mathrm{E}-23$ \\
\hline 10088791 OS & mild/moderate & $1.82 \mathrm{E}-05$ & 0.99998188 \\
\hline 10093928 OS & mild/moderate & $1.98 \mathrm{E}-07$ & 0.99999976 \\
\hline 10117924-20180220@102014-R2 & mild/moderate & 0.99999177 & 8.24E-06 \\
\hline 10131726-20180319@085722-L3 & mild/moderate & 0.007010281 & 0.99298966 \\
\hline 10131726-20180319@085722-R2 & mild/moderate & 0.99999964 & $3.62 \mathrm{E}-07$ \\
\hline 10158969-20180506@090506-L2 & mild/moderate & 0.99999547 & $4.52 \mathrm{E}-06$ \\
\hline 10166525-20180402@083736-L4 & mild/moderate & 0.8872788 & 0.11272123 \\
\hline 10167812-20180424@134719-L2 & mild/moderate & 0.05154828 & 0.94845176 \\
\hline \# of Incorrect Classification & & 16 & 12 \\
\hline
\end{tabular}

\section{$64 \times 64$}

\begin{tabular}{|c|c|c|c|}
\hline Patient & Type & $\begin{array}{l}\text { P (Abnormal) } \\
{[64 * 64]}\end{array}$ & $\begin{array}{l}\mathrm{P}(\text { Normal) } \\
{[64 * 64]}\end{array}$ \\
\hline 10001418 OS & abnormal & 1 & 1.64E-09 \\
\hline 10022953 OS & abnormal & 1 & $8.43 \mathrm{E}-10$ \\
\hline 10029705-20180416@112408-L4 & abnormal & 1 & $8.60 \mathrm{E}-09$ \\
\hline 10029705-20180416@112408-R3 & abnormal & 0.99998713 & $1.29 \mathrm{E}-05$ \\
\hline 10041101 OD & abnormal & 1 & 8.74E-11 \\
\hline 10041101 OS & abnormal & 1 & $1.68 \mathrm{E}-32$ \\
\hline 10046318 OS & abnormal & 1 & $1.55 \mathrm{E}-21$ \\
\hline 10055620 OD & abnormal & 1 & $1.93 \mathrm{E}-20$ \\
\hline 10055620 OS & abnormal & 1 & $2.76 \mathrm{E}-22$ \\
\hline 10065949 OS & abnormal & 0.99999964 & $3.26 \mathrm{E}-07$ \\
\hline
\end{tabular}


10068165 OD
10075046 OD
10075046 OS
10077406 OD
10077406 OS
10084968 OS
10086708 OS
$10117924-20180220 @ 102014-L 4$
$10145754-20180415 @ 105849-L 2$
$10145754-20180415 @ 105849-R 1$
$10148705-20180425 @ 100700-R 3$
$10149811-20180225 @ 090046-R 1$
$10158969-20180506 @ 090506-R 1$
10001091 OD

10001091 OS

10000464 OS

10000484 OD

10001418 OD

10004898 OS

10008285 OS

10013404 OD

10018169 OD

10022953 OD

10022979-20180213@155058-R1

10025329 OD

10028832 OD

10028832 OS

10037058 OD

10037058 OS

10041971 OD

10041971 OS

10047851

10047851 OD

10051334 OD

10051334 OS

10059609-20180325@094033-L3

10065949 OD

10068165 OS

10072783-20180121@092503-L2

10075925 OD

10081192 OS

10084968 OD

10086708 OD

\begin{tabular}{|c|c|c|}
\hline \multirow{2}{*}{$\begin{array}{l}\text { abnormal } \\
\text { abnormal }\end{array}$} & 0.99998391 & $1.62 \mathrm{E}-05$ \\
\hline & 0.99999857 & $1.49 \mathrm{E}-06$ \\
\hline \multirow{2}{*}{$\begin{array}{l}\text { abnormal } \\
\text { abnormal }\end{array}$} & 1 & $4.30 \mathrm{E}-18$ \\
\hline & 1 & $1.64 \mathrm{E}-12$ \\
\hline abnormal & 0.99997497 & $2.51 \mathrm{E}-05$ \\
\hline \multirow{2}{*}{$\begin{array}{l}\text { abnormal } \\
\text { abnormal }\end{array}$} & 1 & $2.28 \mathrm{E}-14$ \\
\hline & 1 & $2.85 \mathrm{E}-15$ \\
\hline abnormal & 0.99986422 & $1.36 \mathrm{E}-04$ \\
\hline abnormal & 0.99998403 & $1.60 \mathrm{E}-05$ \\
\hline abnormal & 1 & $8.69 \mathrm{E}-10$ \\
\hline abnormal & $1.85839 \mathrm{E}-15$ & $1.00 \mathrm{E}+00$ \\
\hline abnormal & 1 & $6.47 \mathrm{E}-14$ \\
\hline & 0.99999356 & $6.40 \mathrm{E}-06$ \\
\hline \multirow{2}{*}{$\begin{array}{l}\text { abnormal } \\
\text { abnormal }\end{array}$} & 1 & 8.70E-14 \\
\hline & 1 & 4.15E-21 \\
\hline mild/moderate & $1.58362 \mathrm{E}-24$ & $1.00 \mathrm{E}+00$ \\
\hline \multirow{2}{*}{$\begin{array}{l}\text { mild/moderate } \\
\text { mild/moderate }\end{array}$} & $1.956 \mathrm{E}-08$ & $1.00 \mathrm{E}+00$ \\
\hline & 5.52701E-09 & $1.00 \mathrm{E}+00$ \\
\hline mild/moderate & $1.45156 \mathrm{E}-40$ & $1.00 \mathrm{E}+00$ \\
\hline mild/moderate & 0.06106123 & 0.9389388 \\
\hline \multirow{2}{*}{$\begin{array}{l}\text { mild/moderate } \\
\text { mild/moderate }\end{array}$} & $1.74044 \mathrm{E}-10$ & 1 \\
\hline & $2.02048 \mathrm{E}-09$ & 1 \\
\hline mild/moderate & $1.57295 \mathrm{E}-14$ & 1 \\
\hline mild/moderate & $1.11 \mathrm{E}-06$ & 0.99999893 \\
\hline mild/moderate & $4.45 \mathrm{E}-11$ & 1 \\
\hline \multirow{2}{*}{ mild/moderate } & 1.11E-06 & 0.99999893 \\
\hline & $2.92 \mathrm{E}-06$ & 0.99999714 \\
\hline $\begin{array}{l}\text { mild/moderate } \\
\text { mild/moderate }\end{array}$ & $1.01 \mathrm{E}-12$ & 1 \\
\hline mild/moderate & $1.00 \mathrm{E}+00$ & $1.16596 \mathrm{E}-11$ \\
\hline mild/moderate & $9.82 \mathrm{E}-01$ & 0.017991316 \\
\hline \multirow{2}{*}{$\begin{array}{l}\text { mild/moderate } \\
\text { mild/moderate }\end{array}$} & 1.79E-08 & 1 \\
\hline & $2.03 \mathrm{E}-10$ & 1 \\
\hline mild/moderate & 4.06E-09 & 1 \\
\hline \multirow{2}{*}{$\begin{array}{l}\text { mild/moderate } \\
\text { mild/moderate }\end{array}$} & $1.91 \mathrm{E}-23$ & 1 \\
\hline & 3.85E-06 & 0.99999619 \\
\hline $\begin{array}{l}\text { mild/moderate } \\
\text { mild/moderate }\end{array}$ & $1.00 \mathrm{E}+00$ & $3.93148 \mathrm{E}-06$ \\
\hline mild/moderate & 8.95E-06 & 0.99999106 \\
\hline mild/moderate & $1.65 \mathrm{E}-05$ & 0.99998355 \\
\hline mild/moderate & $1.07 \mathrm{E}-05$ & 0.99998927 \\
\hline mild/moderate & $1.13 \mathrm{E}-08$ & 1 \\
\hline mild/moderate & $2.30 \mathrm{E}-11$ & 1 \\
\hline mild/moderate & 4.49E-13 & 1 \\
\hline mild/moderate & $1.62 \mathrm{E}-11$ & 1 \\
\hline
\end{tabular}




\begin{tabular}{|c|c|c|c|}
\hline 10088791 OD & mild/moderate & $2.49 \mathrm{E}-05$ & 0.99997509 \\
\hline 10088791 OS & mild/moderate & $0.00 \mathrm{E}+00$ & 1 \\
\hline 10093928 OS & mild/moderate & 4.34E-07 & 0.99999952 \\
\hline 10117924-20180220@102014-R2 & mild/moderate & $5.35 \mathrm{E}-11$ & 1 \\
\hline 10131726-20180319@085722-L3 & mild/moderate & $4.32 \mathrm{E}-07$ & 0.99999952 \\
\hline 10131726-20180319@085722-R2 & mild/moderate & $2.71 \mathrm{E}-11$ & 1 \\
\hline 10158969-20180506@090506-L2 & mild/moderate & 2.51E-05 & 0.99997485 \\
\hline 10166525-20180402@083736-L4 & mild/moderate & $1.07 \mathrm{E}-05$ & 0.99998927 \\
\hline 10167812-20180424@134719-L2 & mild/moderate & $1.01 \mathrm{E}-07$ & 0.99999988 \\
\hline \# of Incorrect Classification & & $2.00 \mathrm{E}+00$ & 0 \\
\hline
\end{tabular}

\section{$128 \times 128$}

\begin{tabular}{|c|c|c|c|}
\hline Patient & Type & $\begin{array}{l}\mathrm{P}(\text { Abnormal) } \\
{[128 * 128]}\end{array}$ & $\begin{array}{l}\mathrm{P}(\text { Normal) } \\
{[128 * 128]} \\
\end{array}$ \\
\hline 10001418 OS & abnormal & 0.99999952 & 4.87E-07 \\
\hline 10022953 OS & abnormal & 0.99995208 & 4.79E-05 \\
\hline 10029705-20180416@112408-L4 & abnormal & 0.99989486 & $1.05 \mathrm{E}-04$ \\
\hline 10029705-20180416@112408-R3 & abnormal & 0.99989188 & 0.000108118 \\
\hline 10041101 OD & abnormal & 0.99984419 & 0.000155748 \\
\hline 10041101 OS & abnormal & 1 & $6.96896 \mathrm{E}-11$ \\
\hline 10046318 OS & abnormal & 0.99984348 & $1.56 \mathrm{E}-04$ \\
\hline 10055620 OD & abnormal & 1 & 8.19795E-10 \\
\hline 10055620 OS & abnormal & 0.9999969 & $3.10 \mathrm{E}-06$ \\
\hline 10065949 OS & abnormal & 0.99955744 & 0.000442587 \\
\hline 10068165 OD & abnormal & 0.99990392 & $9.60951 \mathrm{E}-05$ \\
\hline 10075046 OD & abnormal & 0.99993837 & $6.16 \mathrm{E}-05$ \\
\hline 10075046 OS & abnormal & 0.99998069 & 1.92567E-05 \\
\hline 10077406 OD & abnormal & 0.9999969 & $3.14 \mathrm{E}-06$ \\
\hline 10077406 OS & abnormal & 0.99981743 & 0.000182557 \\
\hline 10084968 OS & abnormal & 0.99996579 & $3.43 \mathrm{E}-05$ \\
\hline 10086708 OS & abnormal & 0.99994266 & $5.73716 \mathrm{E}-05$ \\
\hline 10117924-20180220@102014-L4 & abnormal & 0.99999535 & 4.61176E-06 \\
\hline 10145754-20180415@105849-L2 & abnormal & 0.9999938 & 6.17E-06 \\
\hline 10145754-20180415@105849-R1 & abnormal & 0.9999516 & $4.84 \mathrm{E}-05$ \\
\hline 10148705-20180425@100700-R3 & abnormal & 0.9998908 & 0.000109201 \\
\hline 10149811-20180225@090046-R1 & abnormal & 0.99998224 & $1.77666 \mathrm{E}-05$ \\
\hline 10158969-20180506@090506-R1 & abnormal & 0.99998629 & 1.37E-05 \\
\hline 10001091 OD & abnormal & 0.99988723 & $1.13 \mathrm{E}-04$ \\
\hline 10001091 OS & abnormal & 0.99999547 & $4.55215 \mathrm{E}-06$ \\
\hline 10000464 OS & mild/moderate & 4.67553E-06 & 0.99999535 \\
\hline 10000484 OD & mild/moderate & 0.000164147 & $1.00 \mathrm{E}+00$ \\
\hline
\end{tabular}




\begin{tabular}{|c|c|}
\hline 10001418 OD & mild/modera \\
\hline 0004898 OS & mild/moder \\
\hline 0008285 OS & mild/moder \\
\hline 0013404 OD & mild/mode \\
\hline 10018169 OD & mild/mode \\
\hline 10022953 OD & mild/moder \\
\hline 10022979-20180213@155058-R1 & $\mathrm{mild} / \mathrm{mode}$ \\
\hline 10025329 OD & mild/mode \\
\hline 10028832 OD & mild/mode \\
\hline 10028832 OS & mild/mode \\
\hline 10037058 OD & mild/mode \\
\hline 10037058 OS & $\mathrm{mild} / \mathrm{mode}$ \\
\hline 10041971 OD & $\mathrm{mild} / \mathrm{mode}$ \\
\hline 10041971 OS & mild/moder \\
\hline 10047851 & mild/moder \\
\hline 10047851 OD & mild/moder \\
\hline 10051334 OD & $\mathrm{mild} / \mathrm{mode}$ \\
\hline 10051334 OS & mild/moder \\
\hline 10059609-20180325@094033-L3 & mild/mode \\
\hline 10065949 OD & mild/mode \\
\hline 10068165 OS & $\mathrm{mild} / \mathrm{mod}$ \\
\hline 10072783-20180121@092503-L2 & mild/moder \\
\hline 10075925 OD & mild/moder \\
\hline 10081192 OS & mild/moder \\
\hline 10084968 OD & mild/moder \\
\hline 10086708 OD & mild/moder \\
\hline 10088791 OD & mild/moder \\
\hline 10088791 OS & mild/moder \\
\hline 10093928 OS & mild/moder \\
\hline 10117924-20180220@102014-R2 & mild/moder \\
\hline 10131726-20180319@085722-L3 & mild/moder \\
\hline 10131726-20180319@085722-R2 & mild/moder \\
\hline 10158969-20180506@090506-L2 & mild/mode \\
\hline 10166525-20180402@083736-L4 & mild/mode \\
\hline 10167812-20180424@134719-L2 & mild/mode \\
\hline
\end{tabular}

\begin{tabular}{|c|c|}
\hline 1.82634E-05 & $1.00 \mathrm{E}+0 \mathrm{C}$ \\
\hline $3.41719 \mathrm{E}-12$ & $1.00 \mathrm{E}+0 \mathrm{C}$ \\
\hline $1.72229 \mathrm{E}-06$ & 0.99999833 \\
\hline 5.08921E-05 & 0.9999491 \\
\hline 0.000141378 & 0.99985862 \\
\hline $6.75234 \mathrm{E}-05$ & 0.99993253 \\
\hline $1.48 \mathrm{E}-04$ & 0.99985194 \\
\hline 1.44E-01 & 0.8564226 \\
\hline $8.03106 \mathrm{E}-06$ & 0.99999201 \\
\hline 7.74E-02 & 0.92256796 \\
\hline $2.03 \mathrm{E}-08$ & 1 \\
\hline 0.000249152 & 0.99975079 \\
\hline 4.99E-05 & 0.99995017 \\
\hline 8.07E-05 & 0.9999193 \\
\hline 0.99851722 & 0.001482814 \\
\hline 1.63E-04 & 0.99983692 \\
\hline 4.39E-05 & 0.99995601 \\
\hline 0.000212336 & 0.99978763 \\
\hline $3.41 \mathrm{E}-05$ & 0.99996591 \\
\hline $1.00 \mathrm{E}+00$ & $5.6119 \mathrm{E}-05$ \\
\hline 0.88677871 & 0.11322127 \\
\hline 0.000187775 & 0.9998123 \\
\hline 0.000161167 & 0.99983883 \\
\hline 1.24959E-06 & 0.99999881 \\
\hline $1.11 \mathrm{E}-05$ & 0.99998891 \\
\hline $6.08 \mathrm{E}-07$ & 0.9999994 \\
\hline $1.00 E+00$ & $5.53622 \mathrm{E}-09$ \\
\hline 2.88794E-05 & 0.99997115 \\
\hline 1.25E-04 & 0.99987519 \\
\hline 0.22493188 & 0.7750681 \\
\hline $1.88 \mathrm{E}-04$ & 0.99981159 \\
\hline 2.67E-10 & 1 \\
\hline 0.9999944 & $5.60941 \mathrm{E}-06$ \\
\hline 0.99999702 & 3.02197E-06 \\
\hline 9.99E-01 & 0.000615534 \\
\hline 7 & c \\
\hline
\end{tabular}

\section{$256 \times 256$}

\begin{tabular}{|l|l|l|l|}
\hline & & $\begin{array}{l}P(\text { Abnormal) } \\
{[256 * 256]}\end{array}$ & $\begin{array}{l}\text { (Normal) } \\
{[256 * 256]}\end{array}$ \\
\hline
\end{tabular}




10001418 OS
10022953 OS
$10029705-20180416 @ 112408-L 4$
$10029705-20180416 @ 112408-R 3$
10041101 OD
10041101 OS
10046318 OS
10055620 OD
10055620 OS
10065949 OS
10068165 OD
10075046 OD
10075046 OS
10077406 OD
10077406 OS
10084968 OS
10086708 OS
$10117924-20180220 @ 102014-L 4$
$10145754-20180415 @ 105849-L 2$
$10145754-20180415 @ 105849-R 1$
$10148705-20180425 @ 100700-R 3$
$10149811-20180225 @ 090046-R 1$
$10158969-20180506 @ 090506-R 1$
10001091 OD
10001091 OS
10000464 OS
10000484 OD
10001418 OD
10004898 OS
10008285 OS
10013404 OD
10018169 OD
10022953 OD
$10022979-20180213 @ 155058-R 1$
10025329 OD
10028832 OD
10028832 OS
10037058 OD
10037058 OS
10041971 OD
10041971 OS
10047851
10047851 OD

\begin{tabular}{|c|c|c|}
\hline abnormal & 1 & $9.18 \mathrm{E}-11$ \\
\hline abnormal & 0.99997783 & $2.22 \mathrm{E}-05$ \\
\hline abnormal & 0.99998868 & $1.14 \mathrm{E}-05$ \\
\hline abnormal & 0.99999261 & $7.3702 \mathrm{E}-06$ \\
\hline abnormal & 0.99998462 & $1.53752 \mathrm{E}-05$ \\
\hline abnormal & 1 & $1.09164 \mathrm{E}-08$ \\
\hline abnormal & 0.99999988 & 9.78637E-08 \\
\hline abnormal & 1 & $2.52 \mathrm{E}-11$ \\
\hline abnormal & 0.99998379 & $1.63 \mathrm{E}-05$ \\
\hline abnormal & 0.99996638 & $3.36 \mathrm{E}-05$ \\
\hline abnormal & 0.99999881 & $1.25081 \mathrm{E}-06$ \\
\hline abnormal & 0.99996507 & $3.49861 \mathrm{E}-05$ \\
\hline abnormal & 0.99999797 & $2.01 \mathrm{E}-06$ \\
\hline abnormal & 0.99999964 & $3.7115 \mathrm{E}-07$ \\
\hline abnormal & 0.99999583 & 4.12848E-06 \\
\hline onormal & 0.99999988 & 1.14369E-07 \\
\hline abnormal & 1 & $3.93208 \mathrm{E}-10$ \\
\hline abnormal & 1 & 3.17E-13 \\
\hline abnormal & 1 & $2.96 \mathrm{E}-08$ \\
\hline abnormal & 1 & $4.2894 \mathrm{E}-15$ \\
\hline abnormal & 0.99994671 & $5.32 \mathrm{E}-05$ \\
\hline abnormal & 0.99999869 & $1.30 \mathrm{E}-06$ \\
\hline abnormal & 0.9999795 & $2.05 \mathrm{E}-05$ \\
\hline abnormal & 0.99997497 & $2.51 \mathrm{E}-05$ \\
\hline abnormal & 1 & $9.43 \mathrm{E}-15$ \\
\hline mild/moderate & $1.80009 \mathrm{E}-07$ & $1.00 \mathrm{E}+00$ \\
\hline mild/moderate & 0.9988721 & 0.001127839 \\
\hline mild/moderate & 9.05139E-09 & $1.00 \mathrm{E}+00$ \\
\hline mild/moderate & 5.21877E-11 & $1.00 \mathrm{E}+00$ \\
\hline mild/moderate & 0.58756328 & 0.41243672 \\
\hline mild/moderate & $9.22898 \mathrm{E}-05$ & 0.99990773 \\
\hline mild/moderate & 0.99998701 & $1.29961 \mathrm{E}-05$ \\
\hline mild/moderate & $1.32348 \mathrm{E}-05$ & 0.99998677 \\
\hline mild/moderate & $2.89 \mathrm{E}-05$ & 0.99997103 \\
\hline mild/moderate & 5.43E-09 & 1 \\
\hline mild/moderate & 0.028293183 & 0.97170681 \\
\hline mild/moderate & 9.00074E-09 & 1 \\
\hline mild/moderate & $3.18 \mathrm{E}-14$ & 1 \\
\hline mild/moderate & $6.41 \mathrm{E}-15$ & 1 \\
\hline mild/moderate & $7.89 \mathrm{E}-06$ & 0.99999213 \\
\hline mild/moderate & 2.33375E-07 & 0.99999976 \\
\hline ild/moderate & $7.12 \mathrm{E}-03$ & 0.99288416 \\
\hline nild/moderate & $3.11 \mathrm{E}-05$ & 0.99996889 \\
\hline
\end{tabular}




10051334 OD
10051334 OS
$10059609-20180325 @ 094033-L 3$
10065949 OD
10068165 OS
$10072783-20180121 @ 092503-L 2$
10075925 OD
10081192 OS
10084968 OD
10086708 OD
10088791 OD
10088791 OS
10093928 OS
$10117924-20180220 @ 102014-R 2$
$10131726-20180319 @ 085722-L 3$
$10131726-20180319 @ 085722-R 2$
$10158969-20180506 @ 090506-L 2$
$10166525-20180402 @ 083736-L 4$
$10167812-20180424 @ 134719-L 2$

\begin{tabular}{|c|c|c|}
\hline mild/moderate & 8.69E-06 & 0.9999913 \\
\hline mild/moderate & $2.20107 \mathrm{E}-09$ & 1 \\
\hline mild/moderate & $1.88 \mathrm{E}-10$ & 1 \\
\hline mild/moderate & $4.30446 \mathrm{E}-05$ & 0.99995697 \\
\hline mild/moderate & 0.99974483 & 0.000255199 \\
\hline mild/moderate & $4.13 \mathrm{E}-05$ & 0.99995875 \\
\hline mild/moderate & 9.99E-01 & 0.000701777 \\
\hline mild/moderate & $3.14926 \mathrm{E}-05$ & 0.99996853 \\
\hline mild/moderate & 3.49794E-13 & $1.00 \mathrm{E}+00$ \\
\hline mild/moderate & $1.06 \mathrm{E}-06$ & 0.99999893 \\
\hline mild/moderate & $1.19 \mathrm{E}-21$ & 1 \\
\hline mild/moderate & 9.07903E-06 & 0.99999094 \\
\hline mild/moderate & $9.8197 \mathrm{E}-07$ & 0.99999905 \\
\hline mild/moderate & 0.99951839 & 0.000481647 \\
\hline mild/moderate & $9.93 \mathrm{E}-10$ & 1 \\
\hline mild/moderate & $2.40 \mathrm{E}-18$ & 1 \\
\hline mild/moderate & $9.26337 \mathrm{E}-06$ & 0.9999907 \\
\hline mild/moderate & 3.21697E-05 & 0.99996781 \\
\hline mild/moderate & $9.34 \mathrm{E}-06$ & 0.9999907 \\
\hline & 6 & $\underline{0}$ \\
\hline
\end{tabular}

\section{$512 \times 512$}

\begin{tabular}{|c|c|c|c|}
\hline Patient & Type & $\begin{array}{l}\text { P (Abnormal) } \\
{[512 * 512]}\end{array}$ & $\begin{array}{l}\mathrm{P}(\text { Normal) } \\
{[512 * 512]}\end{array}$ \\
\hline 10001418 OS & abnormal & 0.99936146 & 0.000638496 \\
\hline 10022953 OS & abnormal & 0.9960413 & 0.003958674 \\
\hline 10029705-20180416@112408-L4 & abnormal & 0.99952638 & 4.74E-04 \\
\hline 10029705-20180416@112408-R3 & abnormal & 0.99940002 & $6.00 \mathrm{E}-04$ \\
\hline 10041101 OD & abnormal & 0.97922426 & 0.020775726 \\
\hline 10041101 OS & abnormal & 0.98724443 & 0.01275562 \\
\hline 10046318 OS & abnormal & 0.92326951 & 0.07673049 \\
\hline 10055620 OD & abnormal & 0.9999845 & $1.55044 \mathrm{E}-05$ \\
\hline 10055620 OS & abnormal & 0.9982987 & $1.70 \mathrm{E}-03$ \\
\hline 10065949 OS & abnormal & 0.003065838 & 0.99693418 \\
\hline 10068165 OD & abnormal & 0.97903001 & 0.020969966 \\
\hline 10075046 OD & abnormal & 0.96919304 & 0.030806985 \\
\hline 10075046 OS & abnormal & 0.034994565 & 0.96500546 \\
\hline 10077406 OD & abnormal & 0.96866268 & 0.031337354 \\
\hline 10077406 OS & abnormal & 1.77919E-07 & 0.99999988 \\
\hline 10084968 OS & abnormal & 0.9262225 & 0.073777564 \\
\hline
\end{tabular}




10086708 OS
$10117924-20180220 @ 102014-L 4$
$10145754-20180415 @ 105849-L 2$
$10145754-20180415 @ 105849-R 1$
$10148705-20180425 @ 100700-R 3$
$10149811-20180225 @ 090046-R 1$
$10158969-20180506 @ 090506-R 1$
10001091 OD
10001091 OS
10000464 OS
10000484 OD
10001418 OD
10004898 OS
10008285 OS
10013404 OD
10018169 OD
10022953 OD
$10022979-20180213 @ 155058-R 1$
10025329 OD
10028832 OD
10028832 OS
10037058 OD
10037058 OS
10041971 OD
10041971 OS
10047851
10047851 OD
10051334 OD
10051334 OS
$10059609-20180325 @ 094033-L 3$
10065949 OD
10068165 OS
$10072783-20180121 @ 092503-L 2$
10075925 OD
10081192 OS
10084968 OD
10086708 OD
10088791 OD
10088791 OS
10093928 OS
$10117924-20180220 @ 102014-R 2$
$10131726-20180319 @ 085722-R 2$

\begin{tabular}{|c|c|c|}
\hline abnormal & 0.004060022 & 0.99593997 \\
\hline abnormal & 0.999982 & $1.7998 \mathrm{E}-05$ \\
\hline abnormal & 0.99996924 & $3.07769 \mathrm{E}-05$ \\
\hline abnormal & 0.99999881 & $1.21834 \mathrm{E}-06$ \\
\hline abnormal & 0.99959844 & 0.00040161 \\
\hline abnormal & 0.97366387 & 0.026336132 \\
\hline abnormal & 0.99999619 & $3.78 \mathrm{E}-06$ \\
\hline abnormal & 0.96762341 & 0.032376532 \\
\hline abnormal & 0.99683553 & $3.16 \mathrm{E}-03$ \\
\hline mild/moderate & $6.08433 \mathrm{E}-06$ & 0.99999392 \\
\hline mild/moderate & 0.01140972 & 0.9885903 \\
\hline mild/moderate & 0.084231965 & 0.91576803 \\
\hline mild/moderate & 0.000502195 & 0.99949777 \\
\hline mild/moderate & 0.005196332 & 0.99480361 \\
\hline mild/moderate & 0.15164208 & 0.84835792 \\
\hline mild/moderate & 0.000914671 & 0.99908531 \\
\hline mild/moderate & 0.046061426 & 0.9539386 \\
\hline mild/moderate & $6.56536 \mathrm{E}-06$ & 0.99999344 \\
\hline mild/moderate & $2.68299 \mathrm{E}-08$ & 1 \\
\hline mild/moderate & $1.29917 \mathrm{E}-05$ & 0.99998701 \\
\hline mild/moderate & 0.001329623 & 0.99867034 \\
\hline mild/moderate & $6.37248 \mathrm{E}-08$ & 0.99999988 \\
\hline mild/moderate & $4.54236 \mathrm{E}-09$ & 1 \\
\hline mild/moderate & 0.72162044 & 0.27837956 \\
\hline mild/moderate & 0.000409728 & 0.99959034 \\
\hline mild/moderate & 0.027813982 & 0.97218597 \\
\hline mild/moderate & 0.062140908 & 0.93785918 \\
\hline mild/moderate & 0.16369528 & 0.83630472 \\
\hline mild/moderate & 0.001955796 & 0.99804413 \\
\hline mild/moderate & 1.27724E-08 & 1 \\
\hline mild/moderate & 0.53620446 & 0.46379557 \\
\hline mild/moderate & 0.001630107 & 0.99836987 \\
\hline mild/moderate & 0.004932033 & 0.99506795 \\
\hline mild/moderate & 0.10453955 & 0.89546043 \\
\hline mild/moderate & 0.010845487 & 0.98915452 \\
\hline mild/moderate & 0.33051535 & 0.66948467 \\
\hline mild/moderate & 0.000114546 & 0.99988544 \\
\hline mild/moderate & 0.68852872 & 0.31147122 \\
\hline mild/moderate & 7.09177E-07 & 0.99999928 \\
\hline mild/moderate & 0.001435881 & 0.99856418 \\
\hline mild/moderate & 0.000862961 & 0.9991371 \\
\hline mild/moderate & 0.004823742 & 0.9951762 \\
\hline mild/moderate & 0.000321771 & 0.99967825 \\
\hline
\end{tabular}




\begin{tabular}{|l|l|r|r|} 
10158969-20180506@090506-L2 & mild/moderate & 0.091181904 & 0.90881807 \\
\cline { 4 - 4 } 10166525-20180402@083736-L4 & mild/moderate & 0.11425235 & 0.88574767 \\
\cline { 4 - 5 } 10167812-20180424@134719-L2 & mild/moderate & 0.027037384 & 0.97296256 \\
\hline \# of Incorrect Classification & & 2 & 4 \\
\hline
\end{tabular}




\section{REFERENCES}

1. Wong T. Y., Cheung C. M., Larsen M., Sharma S., Simó R. Diabetic retinopathy. Nature Reviews Disease Primers. 2016;2, p. 16012.

2. Klein, R., Klein, B. E., Moss, S. E., Davis, M. D. \& DeMets, D. L. The Wisconsin epidemiologic study of diabetic retinopathy. II. Prevalence and risk of diabetic retinopathy when age at diagnosis is less than 30 years. Arch. Ophthalmol. 102, 520-526 (1984).

3. Klein, R., Klein, B. E., Moss, S. E., Davis, M. D. \& DeMets, D. L. The Wisconsin epidemiologic study of diabetic retinopathy. III. Prevalence and risk of diabetic retinopathy when age at diagnosis is 30 or more years. Arch. Ophthalmol. 102, 527-532 (1984).

4. Mohamed, Q., Gillies, M. C. \& Wong, T. Y. Management of diabetic retinopathy: a systematic review. JAMA 298, 902-916 (2007). A systematic review of all major trials of treatment options for $D R$.

5. Lumbroso, Bruno, and Marco Rispoli. Diabetic Retinopathy. 1st ed., Jaypee Brothers Medical Publisher, 2015.

6. Klein, R., Klein, B. E., Moss, S. E., Davis, M. D. \& DeMets, D. L. The Wisconsin epidemiologic study of diabetic retinopathy. IV. Diabetic macular edema. Ophthalmology 91, 1464-1474 (1984).

7. Klein, R., Klein, B. E., Moss, S. E. \& Cruickshanks, K. J. The Wisconsin epidemiologic study of diabetic retinopathy: XVII. The 14-year incidence and progression of diabetic retinopathy and associated risk factors in type 1 diabetes. Ophthalmology 105, 1801-1815 (1998).

8. McPherson, Richard, and Matthew Pincus. Henry's Clinical Diagnosis and Management by Laboratory Methods. 22nd ed., Elsevier, 2011.

9. Sim, D. A. et al. Patterns of peripheral retinal and central macula ischemia in diabetic retinopathy as evaluated by ultra-widefield fluorescein angiography. Am. J. Ophthalmol. 158, 144-153 (2014).

10. Wessel, M. M. et al. Peripheral retinal ischaemia, as evaluated by ultra-widefield fluorescein angiography, is associated with diabetic macular oedema. Br. J. Ophthalmol. 96, 694-698 (2012).

11. Javitt, J. C. \& Aiello, L. P. Cost-effectiveness of detecting and treating diabetic retinopathy. Ann. Intern. Med. 124, 164-169 (1996). This paper demonstrates the cost-effectiveness of screening for DR.

12. Singer, D. E., Nathan, D. M., Fogel, H. A. \& Schachat, A. P. Screening for diabetic retinopathy. Ann. Intern. Med. 116, 660-671 (1992).

13. Williams, G. A. et al. Single-field fundus photography for diabetic retinopathy screening: a report by the American Academy of Ophthalmology.

Ophthalmology 111, 1055-1062 (2004).

14. LeCun Y, Bengio Y, Hinton G. 2015. Deep learning. Nature 521, 436-444.

15. Liu S, Cai W, Che H, Pujol S, Kikinis R, Feng D, Fulham MJ. 2015. Multimodal neuroimaging feature learning for multiclass diagnosis of Alzheimer's disease. IEEE Transactions on Biomedical Engineering 62,11321140.

16. Litjens G, Sánchez CI, Timofeeva N, Hermsen M, Nagtegaal I, Kovacs I, 
Hulsbergenvan de Kaa C, Bult P, Van Ginneken B, Van der Laak J. 2016. Deep learning as a tool for increased accuracy and efficiency of histopathological diagnosis. Scientific Reports 6, 26286.

17. Pratt H, Coenen F, Broadbent DM, Harding SP, Zheng Y. 2016 Convolutional neural networks for diabetic retinopathy. Procedia Comp. Sci. 90, 200-205.

18. S. N. Sangeethaa, P. U. Maheswari, "An intelligent model for blood vessel segmentation in diagnosing DR using CNN", J. Med. Syst., vol. 42, no. 10, pp. 175, Oct. 2018.

19. Chandrakumar T, R Kathirvel, 2016, Classifying Diabetic Retinopathy using Deep Learning Architecture, INTERNATIONAL JOURNAL OF ENGINEERING RESEARCH \& TECHNOLOGY (IJERT) Volume 05, Issue 06 (June 2016).

20. "conv2." MathWorks, MathWorks, www.mathworks.com/help/matlab/ref/conv2.html.

21. Ioffe, Sergey, and Christian Szegedy. "Batch normalization: Accelerating deep network training by reducing internal covariate shift."

22. Nair, Vinod, and Geoffrey E. Hinton. "Rectified linear units improve restricted boltzmann machines." In Proceedings of the 27th international conference on machine learning (ICML-10), pp. 807-814. 2010.

23. Nagi, J., F. Ducatelle, G. A. Di Caro, D. Ciresan, U. Meier, A. Giusti, F. Nagi, J. Schmidhuber, L. M. Gambardella. "Max-Pooling Convolutional Neural Networks for Vision-based Hand Gesture Recognition". IEEE International Conference on Signal and Image Processing Applications (ICSIPA2011), 2011.

24. Glorot, Xavier, and Yoshua Bengio. "Understanding the difficulty of training deep feedforward neural networks." In Proceedings of the thirteenth international conference on artificial intelligence and statistics, pp. 249-256. 2010.

25. He, Kaiming, Xiangyu Zhang, Shaoqing Ren, and Jian Sun. "Delving deep into rectifiers: Surpassing human-level performance on imagenet classification." In Proceedings of the IEEE international conference on computer vision, pp. 1026-1034. 2015.

26. Saxe, Andrew M., James L. McClelland, and Surya Ganguli. "Exact solutions to the nonlinear dynamics of learning in deep linear neural networks."

27. Bishop, C. M. Pattern Recognition and Machine Learning. Springer, New York, NY, 2006.

28. Murphy, K. P. Machine Learning: A Probabilistic Perspective. The MIT Press, Cambridge, Massachusetts, 2012.

29. Krizhevsky, Alex, Ilya Sutskever, and Geoffrey E. Hinton. "ImageNet Classification with Deep Convolutional Neural Networks." Advances in neural information processing systems. 2012.

30. X. Li, T. Pang, B. Xiong, W. Liu, P. Liang, T. Wang, "Convolutional neural networks based transfer learning for diabetic retinopathy fundus image classification", Image and Signal Processing BioMedical Engineering and Informatics (CISP-BMEI) 2017 10th International Congress on. IEEE, pp. 1$11,2017$. 
31. "Diabetic Retinopathy Stages." Tipsense.me, tipsense.me/editorz/. 32. "The Benefits of Optomap." Optos, Optos Plc., www.optos.com/en/products/the-benefits-of-optomap/. 